\title{
COLLAPSE OF THE MEAN CURVATURE FLOW FOR EQUIFOCAL SUBMANIFOLDS*
}

\author{
NAOYUKI KOIKE ${ }^{\dagger}$
}

\begin{abstract}
In this paper, we investigate the mean curvature flows having an equifocal submanifold in a symmetric space of compact type and its focal submanifolds as initial data. It is known that an equifocal submanifold of codimension greater than one in an irreducible symmetric space of compact type occurs as a principal orbit of a Hermann action. However, we investigate the flows conceptionally without use of this fact. The investigation is performed by investigating the mean curvature flows having the lifts of the submanifolds to an (infinite dimensional separable) Hilbert space through a Riemannian submersion as initial data .
\end{abstract}

Key words. Mean curvature flow, equifocal submanifold, isoparametric submanifold.

AMS subject classifications. 53C40, 53C 35 .

1. Introduction. Let $f_{t}$ 's $(t \in[0, T))$ be a one-parameter $C^{\infty}$-family of immersions of a manifold $M$ into a Riemannian manifold $N$, where $T$ is a positive constant or $T=\infty$. Define a map $\tilde{f}: M \times[0, T) \rightarrow N$ by $\widetilde{f}(x, t)=f_{t}(x)((x, t) \in M \times[0, T))$. If, for each $t \in[0, T), \widetilde{f}_{*}\left(\left(\frac{\partial}{\partial t}\right)_{(x, t)}\right)$ is the mean curvature vector of $f_{t}: M \hookrightarrow N$, then $f_{t}$ 's $(t \in[0, T))$ is called a mean curvature flow. In particular, if $f_{t}$ 's are embeddings, then we call $M_{t}:=f_{t}(M)$ 's $(0 \in[0, T))$ rather than $f_{t}$ 's $(0 \in[0, T))$ a mean curvature flow. Liu-Terng [LT] investigated the mean curvature flow having isoparametric submanifolds (or their focal submanifolds) in a Euclidean space as initial data and obtained the following facts.

FACT 1 ([LT]). Let $M$ be a compact isoparametric submanifold in a Euclidean space. Then the following statements (i) and (ii) hold:

(i) The mean curvature flow $M_{t}$ having $M$ as initial data collapses to a focal submanifold of $M$ in finite time. If a focal map of $M$ onto $F$ is spherical, then the mean curvature flow $M_{t}$ has type I singularity, that is, $\lim _{t \rightarrow T-0} \max _{v \in S^{\perp} M_{t}}\left\|A_{v}^{t}\right\|_{\infty}^{2}(T-$ $t)<\infty$, where $A_{v}^{t}$ is the shape operator of $M_{t}$ for $v,\left\|A_{v}^{t}\right\|_{\infty}$ is the sup norm of $A_{v}^{t}$ and $S^{\perp} M_{t}$ is the unit normal bundle of $M_{t}$.

(ii) For any focal submanifold $F$ of $M$, there exists a parallel submanifold $M^{\prime}$ of $M$ such that the mean curvature flow having $M^{\prime}$ as initial data collapses to $F$ in finite time.

FACT $2([\mathrm{LT}])$. Let $M$ be as in Fact $1, C$ be the Weyl domain of $M$ at $x_{0}(\in M)$ and $\sigma$ be a stratum of dimension greater than zero of $\partial C$. Then the following statements (i) and (ii) hold:

(i) For any focal submanifold $F$ (of $M$ ) through $\sigma$, the maen curvature flow $F_{t}$ having $F$ as initial data collapses to a focal submanifold $F^{\prime}$ (of $M$ ) through $\partial \sigma$ in finite time. If the fibration of $F$ onto $F^{\prime}$ is spherical, then the mean curvature flow $F_{t}$ has type I singularity.

\footnotetext{
*Received June 14, 2010; accepted for publication December 10, 2010.

$\dagger$ Department of Mathematics, Faculty of Science, Tokyo University of Science, 1-3 Kagurazaka, Shinjuku-ku, Tokyo 162-8601 Japan (koike@ma.kagu.tus.ac.jp).
} 
(ii) For any focal submanifold $F$ (of $M$ ) through $\partial \sigma$, there exists a focal submanifold $F^{\prime}$ (of $M$ ) through $\sigma$ such that the mean curvature flow $F_{t}^{\prime}$ having $F^{\prime}$ as initial data collapses to $F$ in finite time.

As a generalized notion of compact isoparametric hypersurfaces in a sphere and a hyperbolic space, and a compact isoparametric submanifolds in a Euclidean space, Terng-Thorbergsson [TT] defined the notion of an equifocal submanifold in a symmetric space as a compact submanifold $M$ satisfying the following three conditions:

(i) the normal holonomy group of $M$ is trivial,

(ii) $M$ has a flat section, that is, for each $x \in M, \Sigma_{x}:=\exp ^{\perp}\left(T_{x}^{\perp} M\right)$ is totally geodesic and the induced metric on $\Sigma_{x}$ is flat, where $T_{x}^{\perp} M$ is the normal space of $M$ at $x$ and $\exp ^{\perp}$ is the normal exponential map of $M$.

(iii) for each parallel normal vector field $v$ of $M$, the focal radii of $M$ along the normal geodesic $\gamma_{v_{x}}\left(\right.$ with $\left.\gamma_{v_{x}}^{\prime}(0)=v_{x}\right)$ are independent of the choice of $x \in M$, where $\gamma_{v_{x}}^{\prime}(0)$ is the velocity vector of $\gamma_{v_{x}}$ at 0 .

On the other hand, Heintze-Liu-Olmos [HLO] defined the notion of an isoparametric submanifold with flat section in a general Riemannian manifold as a submanifold $M$ satisfying the above condition (i) and the following conditions (ii') and (iii'):

(ii') for each $x \in M$, there exists a neighborhood $U_{x}$ of the zero vector (of $T_{x}^{\perp} M$ ) in $T_{x}^{\perp} M$ such that $\Sigma_{x}:=\exp ^{\perp}\left(U_{x}\right)$ is totally geodesic and the induced metric on $\Sigma_{x}$ is flat,

(iii') sufficiently close parallel submanifolds of $M$ are $\mathrm{CMC}$ with respect to the radial direction.

In the case where the ambient space is a symmetric space $G / K$ of compact type, they showed that the notion of an isoparametric submanifold with flat section coincides with that of an equifocal submanifold. The proof was performed by investigating its lift to $H^{0}([0,1], \mathfrak{g})$ through a Riemannian submersion $\pi \circ \phi$, where $\pi$ is the natural projection of $G$ onto $G / K$ and $\phi$ is the parallel transport map for $G$ (which is a Riemannian submersion of $H^{0}([0,1], \mathfrak{g})$ onto $G(\mathfrak{g}$ :the Lie algebra of $\left.G)\right)$. Let $M$ be an equifocal submanifold in $G / K$ and $v$ be a parallel normal vector field of $M$. The end-point map $\eta_{v}(: M \mapsto G / K)$ for $v$ is defined by $\eta_{v}(x)=\exp ^{\perp}\left(v_{x}\right)(x \in M)$. Set $M_{v}:=\eta_{v}(M)$. We call $M_{v}$ a parallel submanifold of $M$ when $\operatorname{dim} M_{v}=\operatorname{dim} M$ and a focal submanifold of $M$ when $\operatorname{dim} M_{v}<\operatorname{dim} M$. The parallel submanifolds of $M$ are equifocal. Let $f: M \times[0, T) \rightarrow G / K$ be the mean curvature flow having $M$ as initial data. Then, it is shown that, for each $t \in[0, T), f_{t}: M \hookrightarrow G / K$ is a parallel submanifold of $M$ and hence it is equifocal (see Lemma 3.1). Fix $x_{0} \in M$. Let $\widetilde{C}\left(\subset T_{x_{0}}^{\perp} M\right)$ be the fundamental domain containing the zero vector (of $T_{x_{0}}^{\perp} M$ ) of the Coxeter group (which acts on $T_{x_{0}}^{\perp} M$ ) of $M$ at $x_{0}$ and set $C:=\exp ^{\perp}(\widetilde{C})$, where we note that $\left.\exp ^{\perp}\right|_{\widetilde{C}}$ is a diffeomorphism onto $C$. Without loss of generality, we may assume that $G$ is simply connected. Set $\widetilde{M}:=(\pi \circ \phi)^{-1}(M)$, which is an isoparametric submanifold in $H^{0}([0,1], \mathfrak{g})$. Fix $u_{0} \in(\pi \circ \phi)^{-1}\left(x_{0}\right)$. The normal space $T_{x_{0}}^{\perp} M$ is identified with the normal space $T_{u_{0}}^{\perp} \widetilde{M}$ of $\widetilde{M}$ at $u_{0}$ through $(\pi \circ \phi)_{* u_{0}}$. Each parallel submanifold of $M$ intersects with $C$ at the only point and each focal submanifold of $M$ intersects with $\partial C$ at the only point, where $\partial C$ is the boundary of $C$. Hence, for the mean curvature flow $f: M \times[0, T) \rightarrow G / K$ having $M$ as initial data, each $M_{t}\left(:=f_{t}(M)\right)$ intersects with $C$ at the only point. Denote by $x(t)$ this intersection 
point and define $\xi:[0, T) \rightarrow \widetilde{C}\left(\subset T_{x_{0}}^{\perp} M=T_{u_{0}}^{\perp} \widetilde{M}\right)$ by $\exp ^{\perp}(\xi(t))=x(t)(t \in[0, T))$. Set $\widetilde{M}_{t}:=(\pi \circ \phi)^{-1}\left(M_{t}\right)(t \in[0, T))$. It is shown that $\widetilde{M}_{t}(t \in[0, T))$ is the mean curvature flow having $\widetilde{M}$ as initial data because the mean curvature vector of $\widetilde{M}_{t}$ is the horizontal lift of that of $M_{t}$ through $\pi \circ \phi$. By investigating $\xi:[0, T) \rightarrow T_{u_{0}}^{\perp} \widetilde{M}$, we obtain the following fact corresponding to Fact 1.

Theorem A. Let $M$ be an equifocal submanifold in a symmetric space $G / K$ of compact type. Then the following statements (i) and (ii) hold:

(i) If $M$ is not minimal, then the mean curvature flow $M_{t}$ having $M$ as initial data collapses to a focal submanifold $F$ of $M$ in finite time. Furtheremore, if $M$ is irreducible, the codimension of $M$ is greater than one and if the fibration of $M$ onto $F$ is spherical, then the flow $M_{t}$ has type I singularity.

(ii) For any focal submanifold $F$ of $M$, there exists a parallel submanifold of $M$ collapsing to $F$ along the mean curvature flow and the set of all parallel submanifolds collapsing to $F$ along the mean curvature flow is a one-parameter $C^{\infty}$-family.

REMARK 1.1. U. Christ ([Ch]) showed that all irreducible equifocal submanifolds of codimension greater than one on symmetric spaces of compact type are homogeneous and hence they occur as principal orbits of hyperpolar actions. On the other hand, A. Kollross ([Kol] $)$ showed that all hyperpolar actions of cohomogeneity greater than one on irrdeucible symmetric spaces of compact type are orbit equivalent to Hermann actions on the space. Hence all equifocal submanifolds of codimension greater than one on irreducible symmetric spaces of compact type occurs as principal orbits of Hermann actions. Therefore they are classified completely. Hence we can show the statement of Theorem A by using this classification. However, it is very important to prove the statement conceptionally without the use of this classification. In fact, Liu-Terng ([LT]) prove the results in [LT] conceptionally without the use of the classification of isoparametric submanifolds in Euclidean spaces by J. Dadok ([D]). So, in this paper, we prove the statement of Theorem A conceptionally.

Also, we obtain the following fact corresponding to Fact 2 for the mean curvature flow having a focal submanifold of an equifocal submanifold as initial data.

Theorem B. Let $M$ be as in the statement of Theorem $A$ and $\sigma$ be a stratum of dimension greater than zero of $\partial C$ (which is a stratified space). Then the following statements (i) and (ii) hold:

(i) For any non-minimal focal submanifold $F$ (of $M$ ) through $\sigma$, the maen curvature flow $F_{t}$ having $F$ as initial data collapses to a focal submanifold $F^{\prime}$ (of $M$ ) through $\partial \sigma$ in finite time. Furthermore, if $M$ is irreducible, the codimension of $M$ is greater than one and if the fibration of $F$ onto $F^{\prime}$ is spherical, then the flow $F_{t}$ has type I singularity.

(ii) For any focal submanifold $F$ of $M$ through $\partial \sigma$, there exists a focal submanifold of $M$ through $\sigma$ collapsing to $F$ along the mean curvature flow, and the set of all focal submanifolds of $M$ through $\sigma$ collapsing to $F$ along the mean curvature flow is a one-parameter $C^{\infty}$-family.

Since focal submanifolds of $M$ through 0-dimensional stratums of $\partial C$ are minimal, it follows from these theorems that $M$ collapses to a minimal focal submanifold of $M$ after finitely many times of collapses along the mean curvature flows. 


$$
\begin{aligned}
& M_{t} \underset{\left(t \rightarrow T_{1}\right)}{\longrightarrow} \quad \begin{array}{c}
F^{1} \\
\text { non-min. }
\end{array} \\
& F_{t}^{1} \underset{\left(t \rightarrow T_{2}\right)}{\longrightarrow} \underset{\text { non }-\min .}{F^{2}}
\end{aligned}
$$

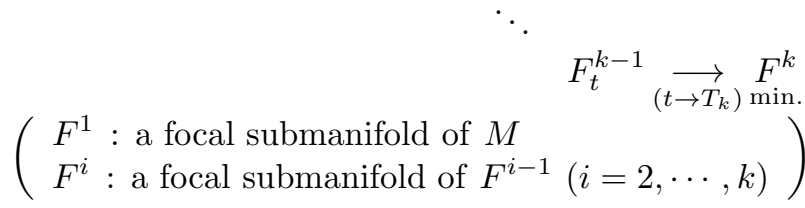

According to the homogeneity theorem for an equifocal submanifold by Christ [Ch], all irreducible equifocal submanifolds of codimension greater than one in symmetric spaces of compact type are homogeneous. Hence, according to the result by HeintzePalais-Terng-Thorbergsson [HPTT], they are principal orbits of hyperpolar actions. Furthermore, according to the classification by Kollross [Kol] of hyperpolar actions on irreducible symmetric spaces of compact type, all hyperpolar actions of cohomogeneity greater than one on the symmetric spaces are Hermann actions. Therefore, all equifocal submanifolds of codimension greater than one in irreducible symmetric spaces of compact type are principal orbits of Hermann actions. In the last section, we describe explicitly the mean curvature flows having orbits of Hermann actions of cohomogeneity two on irreducible symmetric spaces of compact type and rank two as initial data.

2. Preliminaries. In this section, we briefly review the quantities associated with an isoparametric submanifold in an (infinite dimensional separable) Hilbert space, which was introduced by Terng [T2]. Let $M$ be an isoparametric submanifold in a Hilbert space $V$.

2.1. Principal curvatures, curvature normals and curvature distributions. Let $E_{0}$ and $E_{i}(i \in I)$ be all the curvature distributions of $M$, where $E_{0}$ is defined by $\left(E_{0}\right)_{x}=\underset{v \in T_{x}^{\perp} M}{\cap} \operatorname{Ker} A_{v}(x \in M)$. For each $x \in M$, we have $T_{x} M=\overline{\left(E_{0}\right)_{x} \oplus\left(\oplus_{i \in I}\left(E_{i}\right)_{x}\right)}$, which is the common eigenspace decomposition of $A_{v}$ 's $\left(v \in T_{x}^{\perp} M\right)$. Also, let $\lambda_{i}(i \in I)$ be the principal curvatures of $M$, that is, $\lambda_{i}$ is the section of the dual bundle $\left(T^{\perp} M\right)^{*}$ of $T^{\perp} M$ such that $\left.A_{v}\right|_{\left(E_{i}\right)_{x}}=\left(\lambda_{i}\right)_{x}(v)$ id holds for any $x \in M$ and any $v \in T_{x}^{\perp} M$, and $\mathbf{n}_{i}$ be the curvature normal corresponding to $\lambda_{i}$, that is, $\lambda_{i}(\cdot)=\left\langle\mathbf{n}_{i}, \cdot\right\rangle$.

2.2. The Coxeter group associated with an isoparametric submanifold. Denote by $l_{i}^{x}$ the affine hyperplane $\left(\lambda_{i}\right)_{x}^{-1}(1)$ in $T_{x}^{\perp} M$. The focal set of $M$ at $x$ is equal to the sum $\cup_{i \in I}\left(x+l_{i}^{x}\right)$ of the affine hyperplanes $x+l_{i}^{x}$ s $(i \in I)$ in the affine subspace $x+T_{x}^{\perp} M$ of $V$. Each affine hyperplane $l_{i}^{x}$ is called a focal hyperplane of $M$ at $x$. Let $W$ be the group generated by the reflection $R_{i}^{x}$ 's $(i \in I)$ with respect to $l_{i}^{x}$. This group is independent of the choice $x$ of $M$ up to group isomorphism. This group is called the Coxeter group associated with $M$. The fundamental domain of the Coxeter group containing the zero vector of $T_{x}^{\perp} M$ is given by $\left\{v \in T_{x}^{\perp} M \mid \lambda_{i}(v)<1(i \in I)\right\}$.

2.3. Principal curvatures of parallel submanifolds. Let $M_{w}$ be the parallel submanifold of $M$ for a (non-focal) parallel normal vector field $w$, that is, $M_{w}=$ 
$\eta_{w}(M)$, where $\eta_{w}$ is the end-point map for $w$. Denote by $A^{w}$ the shape tensor of $M_{w}$. This submanifold $M_{w}$ also is isoparametric and $\left.A_{v}^{w}\right|_{\eta_{w *}\left(E_{i}\right)_{x}}=\frac{\left(\lambda_{i}\right)_{x}(v)}{1-\left(\lambda_{i}\right)_{x}\left(w_{x}\right)} \mathrm{id}(i \in I)$ for any $v \in T_{\eta_{w}(x)}^{\perp} M_{w}$, that is, $\frac{\lambda_{i}}{1-\lambda_{i}(w)}$ 's $(i \in I)$ are the principal curvatures of $M_{w}$ and hence $\frac{\mathbf{n}_{i}}{1-\lambda_{i}(w)}$ 's $(i \in I)$ are the curvature normals of $M_{w}$, where we identify $T_{\eta_{w}(x)}^{\perp} M_{w}$ with $T_{x}^{\perp} M$.

Let $M$ be a (general) submanifold in a Hilbert space $V$.

2.4. The mean curvature vector of a regularizable submanifold. Assume that $M$ is regularizable in sense of [HLO], that is, for each normal vector $v$ of $M$, the regularizable trace $\operatorname{Tr}_{r} A_{v}$ and $\operatorname{Tr} A_{v}^{2}$ exist, where $\operatorname{Tr}_{r} A_{v}$ is defined by $\operatorname{Tr}_{r} A_{v}:=$ $\sum_{i=1}^{\infty}\left(\mu_{i}^{+}+\mu_{i}^{-}\right)\left(\mu_{1}^{-} \leq \mu_{2}^{-} \leq \cdots \leq 0 \leq \cdots \leq \mu_{2}^{+} \leq \mu_{1}^{+}\right.$: the spectrum of $\left.A_{v}\right)$. Then the mean curvature vector $H$ of $M$ is defined by $\langle H, v\rangle=\operatorname{Tr}_{r} A_{v}\left(\forall v \in T^{\perp} M\right)$.

2.5. The mean curvature flow for a regularizable submanifold. Let $f_{t}: M \hookrightarrow V(0 \leq t<T)$ be a $C^{\infty}$-family of regularizable submanifold immersions into $V$. Denote by $H_{t}$ the mean curvature vector of $f_{t}$. Define a map $F: M \times[0, T) \rightarrow V$ by $F(x, t):=f_{t}(x)((x, t) \in M \times[0, T))$. If $\frac{\partial F}{\partial t}=H_{t}$ holds, then we call $f_{t}(0 \leq$ $t<T)$ the mean curvature flow. In particular, if $f_{t}$ 's are embeddings, then we set $M_{t}:=f_{t}(M)$ and call $M_{t}$ (rather than $f_{t}$ ) the mean curvature flow. Note that, for a given regularizable submanifold immersion $f: M \hookrightarrow V$, there does not necessarily exist the mean curvature flow having $f$ as initial data in short time. Furthermore, we note that, for the restriction $\left.f\right|_{U}$ of $f$ to a relative compact domain $U$ of $M$, the existence of the mean curvature flow having $\left.f\right|_{U}$ as initial data in short time is not assured for the sake of absence of the infinite dimensional vector bundle version of the Hamilton's theorem (Theorem 5.1 of [Ha]) for the existence and the uniqueness of solutions of a certain kind of evolution equation (which includes the Ricci flow equation and the mean curvature flow equation (of finite dimensional case)) in short time.

Let $M$ be an equifocal submanifold in a symmetric space $G / K$ of compact type and set $\widetilde{M}:=(\pi \circ \phi)^{-1}(M)$, where $\pi$ is the natural projection of $G$ onto $G / K$ and $\phi: H^{0}([0,1], \mathfrak{g}) \rightarrow G$ is the parallel transport map for $G$.

2.6. The mean curvature vector of the lifted submanifold. Denote by $\widetilde{H}$ (resp. $H$ ) the mean curvature vector of $\widetilde{M}$ (resp. $M$ ). Then $\widetilde{M}$ is a regularizable isoparametric submanifold and $\widetilde{H}$ is equal to the horizontal lift of $H^{L}$ of $H$ (see Lemma 5.2 of $[\mathrm{HLO}])$.

3. Proofs of Theorems A and B. In this section, we prove Theorems A and B. Let $M$ be an equifocal submanifold in a symmetric space $G / K$ of compact type, $\pi: G \rightarrow G / K$ be the natural projection and $\phi$ be the parallel transport map for $G$. Set $\widetilde{M}:=(\pi \circ \phi)^{-1}(M)$. Take $u_{0} \in \widetilde{M}$ and set $x_{0}:=(\pi \circ \phi)\left(u_{0}\right)$. We identify $T_{x_{0}}^{\perp} M$ with $T_{u_{0}}^{\perp} \widetilde{M}$. Let $\widetilde{C}\left(\subset T_{u_{0}}^{\perp} \widetilde{M}=T_{x_{0}}^{\perp} M\right)$ be the fundamental domain of the Coxeter group of $\widetilde{M}$ at $u_{0}$ containing the zero vector $\mathbf{0}$ of $T_{u_{0}}^{\perp} \widetilde{M}\left(=T_{x_{0}}^{\perp} M\right)$ and set $C:=\exp ^{\perp}(\widetilde{C})$, where $\exp ^{\perp}$ is the normal exponential map of $M$. Denote by $H$ (resp. $\widetilde{H})$ the mean curvature vector of $M$ (resp. $\widetilde{M})$. The mean curvature vector $H$ and $\widetilde{H}$ are a parallel normal vector field of $M$ and $\widetilde{M}$, respectively. Let $w$ be a parallel 
normal vector field of $M$ and $w^{L}$ be the horizontal lift of $w$ to $H^{0}([0,1], \mathfrak{g})$, which is a parallel normal vector field of $\widetilde{M}$. Denote by $M_{w}$ (resp. $\widetilde{M}_{w^{L}}$ ) the parallel (or focal) submanifold $\eta_{w}(M)$ (resp. $\eta_{w^{L}}(\widetilde{M})$ ) of $M$ (resp. $\left.\widetilde{M}\right)$, where $\eta_{w}$ (resp. $\eta_{w^{L}}$ ) is the end-point map for $w$ (resp. $\left.w^{L}\right)$. Then we have $\widetilde{M}_{w^{L}}=(\pi \circ \phi)^{-1}\left(M_{w}\right)$. Denote by $H^{w}$ (resp. $\left.\widetilde{H}^{w^{L}}\right)$ the mean curvature vector of $M_{w}$ (resp. $\left.\widetilde{M}_{w^{L}}\right)$. Define a vector field $X$ on $\widetilde{C}\left(\subset T_{u_{0}}^{\perp} \widetilde{M}=T_{x_{0}}^{\perp} M\right)$ by $X_{w}:=\left(\widetilde{H}^{\widetilde{w}}\right)_{u_{0}+w}(w \in \widetilde{C})$, where $\widetilde{w}$ is the parallel normal vector field of $\widetilde{M}$ with $\widetilde{w}_{u_{0}}=w$. Let $\xi:(-S, T) \rightarrow \widetilde{C}$ be the maximal integral curve of $X$ with $\xi(0)=\mathbf{0}$. Note that $S$ and $T$ are possible be equal to $\infty$. Let $\widetilde{\xi(t)}$ be the parallel normal vector field of $M$ with $\widetilde{\xi(t)} x_{x_{0}}=\xi(t)$.

LEMmA 3.1. The family $\widetilde{M}_{\widetilde{\xi(t)}^{L}}(0 \leq t<T)$ is the mean curvature flow having $\widetilde{M}$ as initial data and $M_{\widetilde{\xi(t)}}(0 \leq t<T)$ is the mean curvature flow having $M$ as initial data.

Proof. Fix $t_{0} \in[0, T)$. Define a flow $F: \widetilde{M} \times[0, T) \rightarrow H^{0}([0,1], \mathfrak{g})$ by $F(u, t):=$ $\eta_{\widetilde{\xi(t)}}{ }_{L}(u)((u, t) \in \widetilde{M} \times[0, T))$ and $f_{t}: \widetilde{M} \rightarrow H^{0}([0,1], \mathfrak{g})(0 \leq t<T)$ by $f_{t}(u):=$ $F(u, t)(u \in \widetilde{M})$. Here we note that $f_{t}(\widetilde{M})=\widetilde{M}_{\widetilde{\xi(t)}}^{L}$. For simplicity, denote by $\widetilde{H}^{t_{0}}$ the mean curvature vector of $\widetilde{M}_{{\widetilde{\xi\left(t t_{0}\right)}}^{L}}$. It is easy to show that $F_{*}\left(\left(\frac{\partial}{\partial t}\right)_{\left(\cdot, t_{0}\right)}\right)$ is a parallel normal vector field of $\widetilde{M_{\tilde{\xi\left(t_{0}\right)}}}{ }^{L}$ and that $F_{*}\left(\left(\frac{\partial}{\partial t}\right)_{\left(u_{0}, t_{0}\right)}\right)=\left(\widetilde{H}^{t_{0}}\right)_{f_{t_{0}}\left(u_{0}\right)}$. On the other hand, since $\widetilde{M}_{\widetilde{\xi\left(t_{0}\right)}}{ }^{L}$ is isoparametric, $\widetilde{H}^{t_{0}}$ is also a parallel normal vector field of $\widetilde{M}_{\widetilde{\xi\left(t_{0}\right)}} L$. Hence we have $F_{*}\left(\left(\frac{\partial}{\partial t}\right)_{\left(\cdot, t_{0}\right)}\right)=\widetilde{H}^{t_{0}}$. Therefore, it follows from the arbitrariness of $t_{0}$ that $\widetilde{M}_{\widetilde{\xi(t)}^{L}}(0 \leq t<T)$ is the mean curvature flow having $\widetilde{M}$ as initial data. Define a flow $\bar{F}: M \times[0, T) \rightarrow G / K$ by $\bar{F}(x, t):=\eta_{\widetilde{\xi(t)}}(x)$ $((x, t) \in M \times[0, T))$ and $\bar{f}_{t}: M \rightarrow G / K(0 \leq t<T)$ by $\bar{f}_{t}(x):=\bar{F}(x, t)(x \in M)$. Here we note that $\bar{f}_{t}(M)=M_{\widetilde{\xi(t)}}$. For simplicity, denote by $H^{t}$ the mean curvature vector of $M_{\widetilde{\xi(t)}}$. Fix $t_{0} \in[0, T)$. Since $\widetilde{M}_{\widetilde{\xi\left(t_{0}\right)}}{ }^{L}=(\pi \circ \phi)^{-1}\left(M_{\widetilde{\xi\left(t_{0}\right)}}\right)$, we have $\left(H^{t_{0}}\right)^{L}=\widetilde{H}^{t_{0}}$. On the other hand, we have $\bar{F}_{*}\left(\left(\frac{\partial}{\partial t}\right)_{\left(\cdot, t_{0}\right)}\right)^{L}=F_{*}\left(\left(\frac{\partial}{\partial t}\right)_{\left(\cdot, t_{0}\right)}\right)\left(=\widetilde{H}^{t_{0}}\right)$. Hence we have $\bar{F}_{*}\left(\left(\frac{\partial}{\partial t}\right)_{\left(\cdot, t_{0}\right)}\right)=H^{t_{0}}$. Therefore, it follows from the arbitrariness of $t_{0}$ that $M_{\widetilde{\xi(t)}}$ $(0 \leq t<T)$ is the mean curvature flow having $M$ as initial data.

Proof of Theorem A. Clearly we suffice to show the statement of Theorem A in the case where $M$ is full. Hence, in the sequel, we assume that $M$ is full. Denote by $\Lambda$ the set of all principal curvatures of $\widehat{M}$. Set $r:=\operatorname{codim} M$. It is shown that the set of all focal hyperplanes of $\widetilde{M}$ is given as the sum of finite pieces of infinite parallel families consisting of hyperplanes in $T_{u_{0}}^{\perp} \widetilde{M}$ which arrange at equal intervals. Let $\left\{l_{a j} \mid j \in \mathbb{Z}\right\} \quad(1 \leq a \leq \bar{r})$ be the finite pieces of infinite parallel families consisting of hyperplanes in $T_{u_{0}}^{\perp} \widetilde{M}$. Since $l_{a j}$ 's $(j \in \mathbb{Z})$ arrange at equal intervals, we can express as $\Lambda=\bigcup_{a=1}^{\bar{r}}\left\{\frac{\lambda_{a}}{1+b_{a} j} \mid j \in \mathbf{Z}\right\}$, where $\lambda_{a}$ 's and $b_{a}$ 's are parallel sections of $\left(T^{\perp} \widetilde{M}\right)^{*}$ and positive constants greater than one, respectively, which are defined by $\left(\left(\lambda_{a}\right)_{u_{0}}\right)^{-1}\left(1+b_{a} j\right)=l_{a j}$. For simplicity, we set $\lambda_{a j}:=\frac{\lambda_{a}}{1+b_{a} j}$. Denote by $\mathbf{n}_{a j}$ and $E_{a j}$ the curvature normal and the curvature distribution corresponding to $\lambda_{a j}$, respectively. The fundamental domain $\widetilde{C}$ of the Coxeter group of $\widetilde{M}$ at $u_{0}$ is given 
by

$$
\widetilde{C}=\left\{w \in T_{u_{0}}^{\perp} \widetilde{M} \mid \lambda_{a}(w)<1(1 \leq a \leq \bar{r})\right\}
$$

It is shown that, for each $a, \lambda_{a, 2 j}$ 's $(j \in \mathbb{Z})$ have the same multiplicity and so are also $\lambda_{a, 2 j+1}$ 's $(j \in \mathbb{Z})$. Denote by $m_{a}^{e}$ and $m_{a}^{o}$ the multiplicities of $\lambda_{a, 2 j}$ and $\lambda_{a, 2 j+1}$, respectively. Take a parallel normal vector field $w$ of $\widetilde{M}$ with $w_{u_{0}} \in \widetilde{C}$. Denote by $\widetilde{A}^{w}$ (resp. $\widetilde{H}^{w}$ ) the shape tensor (resp. the mean curvature vector) of the parallel submanifold $\widetilde{M}_{w}$. Since $\left.\widetilde{A}_{v}^{w}\right|_{\eta_{w *}\left(E_{a j}\right)_{u}}=\frac{\left(\lambda_{a j}\right)_{u}(v)}{1-\left(\lambda_{a j}\right)_{u}\left(w_{u}\right)}$ id $\left(v \in T_{u}^{\perp} \widetilde{M}\right)$, we have

$$
\begin{aligned}
\operatorname{Tr}_{r} \widetilde{A}_{v}^{w} & =\sum_{a=1}^{\bar{r}}\left(\sum_{j \in \mathbf{Z}} \frac{m_{a}^{e}\left(\lambda_{a, 2 j}\right)_{u}(v)}{1-\left(\lambda_{a, 2 j}\right)_{u}\left(w_{u}\right)}+\sum_{j \in \mathbf{Z}} \frac{m_{a}^{o}\left(\lambda_{a, 2 j+1}\right)_{u}(v)}{1-\left(\lambda_{a, 2 j+1}\right)_{u}\left(w_{u}\right)}\right) \\
& =\sum_{a=1}^{\bar{r}}\left(m_{a}^{e} \cot \frac{\pi}{2 b_{a}}\left(1-\left(\lambda_{a}\right)_{u}\left(w_{u}\right)\right)-m_{a}^{o} \tan \frac{\pi}{2 b_{a}}\left(1-\left(\lambda_{a}\right)_{u}\left(w_{u}\right)\right)\right) \frac{\pi}{2 b_{a}}\left(\lambda_{a}\right)_{u}(v),
\end{aligned}
$$

where we use the relation $\cot \frac{\theta}{2}=\sum_{j \in \mathbf{Z}} \frac{2}{\theta+2 j \pi}$. Therefore we have

$$
\widetilde{H}^{w}=\sum_{a=1}^{\bar{r}}\left(m_{a}^{e} \cot \frac{\pi}{2 b_{a}}\left(1-\lambda_{a}(w)\right)-m_{a}^{o} \tan \frac{\pi}{2 b_{a}}\left(1-\lambda_{a}(w)\right)\right) \frac{\pi}{2 b_{a}} \mathbf{n}_{a}
$$

where $\mathbf{n}_{a}$ is the curvature normal corresponding to $\lambda_{a}$. Denote by $\widetilde{\sigma}_{a}(1 \leq a \leq \bar{r})$ the maximal dimensional stratum $\left(\lambda_{a}\right)_{u_{0}}^{-1}(1)$ of $\partial \widetilde{C}$. Fix $a_{0} \in\{1, \cdots, \bar{r}\}$. Take $w_{0} \in \widetilde{\sigma}_{a_{0}}$ and and $w_{0}^{\prime} \in \widetilde{C}$ near $w_{0}$ such that $w_{0}-w_{0}^{\prime}$ is normal to $\widetilde{\sigma}_{a_{0}}$. Set $w_{0}^{\varepsilon}:=\varepsilon w_{0}^{\prime}+(1-\varepsilon) w_{0}$ for $\varepsilon \in(0,1)$. Then we have $\lim _{\varepsilon \rightarrow+0}\left(\lambda_{a_{0}}\right)_{u_{0}}\left(w_{0}^{\varepsilon}\right)=1$ and $\sup _{0<\varepsilon<1}\left(\lambda_{a}\right)_{u_{0}}\left(w_{0}^{\varepsilon}\right)<1$ for each $a \in\{1, \cdots, \bar{r}\} \backslash\left\{a_{0}\right\}$. Hence we have $\lim _{\varepsilon \rightarrow+0} \cot \frac{\pi}{2 b_{a_{0}}}\left(1-\left(\lambda_{a_{0}}\right)_{u_{0}}\left(w_{0}^{\varepsilon}\right)\right)=\infty$ and $\sup _{0<\varepsilon<1} \cot \left(1-\left(\lambda_{a}\right)_{u_{0}}\left(w_{0}^{\varepsilon}\right)\right)<\infty\left(a \in\{1, \cdots, \bar{r}\} \backslash\left\{a_{0}\right\}\right)$. Therefore, we have $\lim _{\varepsilon \rightarrow+0} \frac{X_{w_{0}^{\varepsilon}}}{\left\|X_{w_{0}^{\varepsilon}}^{\varepsilon}\right\|}$ is the outward unit normal vector of $\widetilde{\sigma}_{a_{0}}$. Also we have $\lim _{\varepsilon \rightarrow+0}\left\|X_{w_{0}^{\varepsilon}}\right\|=\infty$. From these facts, $X$ is as in Fig. 1 on a sufficiently small collar neighborhood of $\widetilde{\sigma}_{a_{0}}$.

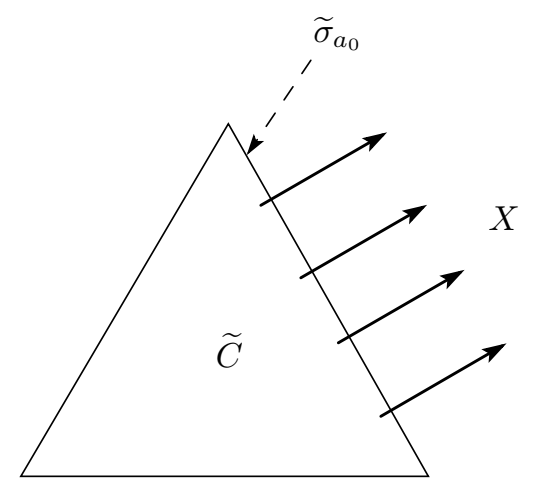

FIG. 1. 
Define a function $\rho$ over $\widetilde{C}$ by

$$
\begin{aligned}
\rho(w):=-\sum_{a=1}^{\bar{r}}( & m_{a}^{e} \log \sin \frac{\pi}{2 b_{a}}\left(1-\left(\lambda_{a}\right)_{u_{0}}(w)\right) \\
& \left.+m_{a}^{o} \log \cos \frac{\pi}{2 b_{a}}\left(1-\left(\lambda_{a}\right)_{u_{0}}(w)\right)\right) \quad(w \in \widetilde{C}) .
\end{aligned}
$$

Let $\left(x_{1}, \cdots, x_{r}\right)$ be the Euclidean coordinate of $T_{u_{0}}^{\perp} \widetilde{M}$. For simplicity, set $\partial_{i}:=\frac{\partial}{\partial x_{i}}$ $(i=1, \cdots, r)$. Then it follows from the definition of $X$ and $(3.1)$ that $\left(\partial_{i} \rho\right)(w) \stackrel{\partial x_{i}}{=}$ $\left\langle X_{w}, \partial_{i}\right\rangle(w \in \widetilde{C}, i=1, \cdots, r)$, that is, $\operatorname{grad} \rho=X$. Also we have

$$
\begin{gathered}
\left(\partial_{i} \partial_{j} \rho\right)(w)=\sum_{a=1}^{\bar{r}}\left(\frac{m_{a}^{e}}{\sin ^{2} \frac{\pi}{2 b_{a}}\left(1-\left(\lambda_{a}\right)_{u_{0}}(w)\right)}+\frac{m_{a}^{o}}{\cos ^{2} \frac{\pi}{2 b_{a}}\left(1-\left(\lambda_{a}\right)_{u_{0}}(w)\right)}\right) \\
\times \frac{\pi^{2}}{4 b_{a}^{2}}\left(\lambda_{a}\right)_{u_{0}}\left(\partial_{i}\right)\left(\lambda_{a}\right)_{u_{0}}\left(\partial_{j}\right) .
\end{gathered}
$$

It follows from this relation that $\rho$ is downward convex. Also it is shown that $\rho(w) \rightarrow$ $\infty$ as $w \rightarrow \partial \widetilde{C}$. Hence we see that $\rho$ has the only minimal point. Denote by $w_{0}$ this minimal point. It is clear that $X_{w_{0}}=0$. From these facts and the fact that $X$ is as in Fig. 1 on a sufficiently small collar neighborhood of each maximal dimensional stratum $\widetilde{\sigma}$ of $\partial \widetilde{C}, \rho$ and $X$ are as in Fig. 2.
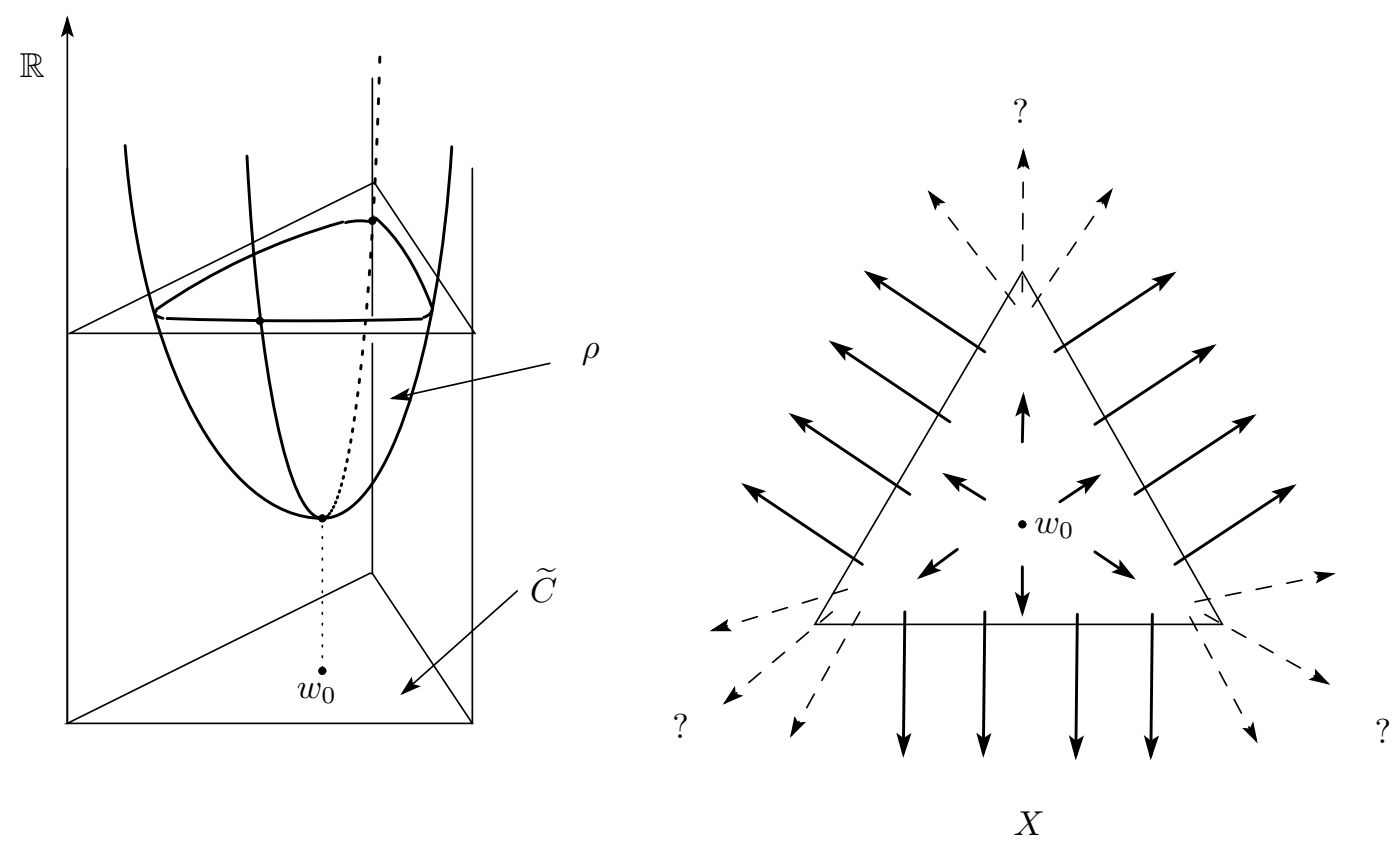

FIG. 2.

Let $W$ be the Coxeter group of $\widetilde{M}$ at $u_{0}$ and $W_{0}$ the isotropy group of $W$ at $\mathbf{0}$. Also, let $\Gamma$ be the lattice of translations of $W$ and set $\Gamma^{*}:=\left\{\omega \in\left(T_{u_{0}}^{\perp} \widetilde{M}\right)^{*} \mid \omega(\Gamma) \subset \mathbf{Z}\right\}$. Also, let $C^{\triangle}\left(T_{u_{0}}^{\perp} \widetilde{M}\right)^{W}$ be the space of all finite sums of $W$-invariant eigenfunctions 
of $\triangle$, where $\triangle$ is the Laplace operator of $T_{u_{0}}^{\perp} \widetilde{M}$. Then we can show

$$
C^{\triangle}\left(T_{u_{0}}^{\perp} \widetilde{M}\right)^{W} \otimes \mathbb{C}=\left\{f=\sum_{\omega \in \Gamma^{*}} a_{\omega} e^{2 \pi \sqrt{-1} \omega} \mid a_{\omega} \in \mathbb{C}\left(a_{\omega}=0 \text { except for finite } \omega\right)\right.
$$

$\& f: W-$ invariant $\}.$

Hence, according to Chapter VI, $\S 3$, Theorem 1 of [B], we have $C^{\triangle}\left(T_{u_{0}}^{\perp} \widetilde{M}\right)^{W} \otimes \mathbb{C}=$ $\mathbb{C}\left[\phi_{1}, \cdots, \phi_{r}\right]$ (polynomial ring) for some $\phi_{1}, \cdots, \phi_{r}\left(\in C^{\triangle}\left(T_{u_{0}}^{\perp} \widetilde{M}\right)^{W}\right)$. Here we note that $r=\operatorname{dim} T_{u_{0}}^{\perp} \widetilde{M}$ because $G / K$ is irreducible and hence $M$ is full and irreducible. By reordering $\phi_{1}, \cdots, \phi_{r}$ suitably, it is shown that $\left\{\operatorname{Re} \phi_{1}, \cdots, \operatorname{Re} \phi_{r_{1}+r_{2}}, \operatorname{Im} \phi_{r_{1}+1}\right.$, $\left.\cdots, \operatorname{Im} \phi_{r_{1}+r_{2}}\right\}$ is a base of $C^{\triangle}\left(T_{u_{0}}^{\perp}(\widetilde{M})\right)^{W}$ (see proof of Theorem 7.6 of [HLO]), where $\operatorname{Re}(\cdot)(\operatorname{resp} . \operatorname{Im}(\cdot))$ is the real part (resp. the imaginary part) of $(\cdot)$, and $r_{1}$ and $r_{2}$ are positive integers with $r_{1}+2 r_{2}=r$. Denote by $\left\{\psi_{1}, \cdots, \psi_{r}\right\}$ this base for simplicity. Set $\Psi:=\left(\psi_{1}, \cdots, \psi_{r}\right)$, which is a $C^{\infty}$-map from $T_{u_{0}}^{\perp} \widetilde{M}$ onto $\mathbb{R}^{r}$. It is shown that $\Psi$ is injective and that $\left.\Psi\right|_{\widetilde{C}}$ is a homeomorphism of $\overline{\widetilde{C}}$ onto $\Psi(\overline{\widetilde{C}})$, where $\overline{\widetilde{C}}$ is the closure of $\widetilde{C}$. Set $\xi_{w}(t):=\psi_{t}(w)$ and $\bar{\xi}_{w}(t):=\Psi\left(\psi_{t}(w)\right)$, where $w \in \widetilde{C}$ and $\left\{\psi_{t}\right\}$ is the local one parameter transformation group of $X$. Also, we set $\xi_{w}^{i}(t):=x_{i}\left(\xi_{w}(t)\right)$ and $\bar{\xi}_{w}^{i}(t):=y_{i}\left(\bar{\xi}_{w}(t)\right)$, where $\left(y_{1}, \cdots, y_{r}\right)$ is the natural coordinate of $\mathbb{R}^{r}$. Then we have

$$
\begin{aligned}
& \left(\bar{\xi}_{w}^{i}\right)^{\prime}(t)=\left\langle\operatorname{grad}\left(y_{i} \circ \Psi\right)_{\xi_{w}(t)}, X_{\xi_{w}(t)}\right\rangle \\
& =\sum_{a=1}^{\bar{r}}\left(\left(m_{a}^{e} \cot \left(\frac{\pi}{2 b_{a}}\left(1-\left(\lambda_{a}\right)_{u_{0}}\left(\xi_{w}(t)\right)\right)\right)-m_{a}^{o} \tan \left(\frac{\pi}{2 b_{a}}\left(1-\left(\lambda_{a}\right)_{u_{0}}\left(\xi_{w}(t)\right)\right)\right)\right)\right. \\
& \left.\quad \times \frac{\pi}{2 b_{a}}\left(\lambda_{a}\right)_{u_{0}}\left(\operatorname{grad}\left(y_{i} \circ \Psi\right)_{\xi_{w}(t)}\right)\right) .
\end{aligned}
$$

Let $f_{i}$ be the $W$-invariant $C^{\infty}$-function over $T_{u_{0}}^{\perp} \widetilde{M}$ such that

$$
\begin{aligned}
f_{i}(v):=\sum_{a=1}^{\bar{r}} & \left(\left(m_{a}^{e} \cot \left(\frac{\pi}{2 b_{a}}\left(1-\left(\lambda_{a}\right)_{u_{0}}(v)\right)\right)-m_{a}^{o} \tan \left(\frac{\pi}{2 b_{a}}\left(1-\left(\lambda_{a}\right)_{u_{0}}(v)\right)\right)\right)\right. \\
& \left.\times \frac{\pi}{2 b_{a}}\left(\lambda_{a}\right)_{u_{0}}\left(\operatorname{grad}\left(y_{i} \circ \Psi\right)_{v}\right)\right)
\end{aligned}
$$

for all $v \in W \cdot \widetilde{C}$. It is easy to show that such a $W$-invariant $C^{\infty}$-function exists uniquely. Hence, we can describe $f_{i}$ as $f_{i}=Y_{i} \circ \Psi$ in terms of some $C^{\infty}$-function $Y_{i}$ over $\mathbb{R}^{r}$. Set $Y:=\left(Y_{1}, \cdots, Y_{r}\right)$, which is regarded as a $C^{\infty}$-vector field on $\mathbb{R}^{r}$. Then we have $Y_{\Psi(w)}=\Psi_{*}\left(X_{w}\right)(w \in \widetilde{C})$, that is, $\left.Y\right|_{\Psi(\widetilde{C})}=\Psi_{*}(X)$. Also we can show that $\left.Y\right|_{\partial \Psi(\widetilde{C})}$ has no zero point. From these facts and the fact that $X$ is as in Fig. 2 , we see that the flow of $X$ starting any point of $\widetilde{C}$ other than $w_{0}\left(w_{0}\right.$ : the only zero point of $X$ ) converges to a point of $\partial \widetilde{C}$ in finite time, and that, furthermore, for each point of $\partial \widetilde{C}$, there exists a unique flow of $X$ converging to the point. Since $M$ is not minimal by the assumption, we have $X_{\mathbf{0}} \neq 0$, that is, $\mathbf{0} \neq w_{0}$. Hence we have $T<\infty$ and $\lim _{t \rightarrow T} \xi(t) \in \partial \widetilde{C}$, where $\xi(t)=\psi_{t}(\mathbf{0})$ and $T$ is the supremum of the domain of $\xi$. Set $w_{1}:=\lim _{t \rightarrow T-0} \xi(t)$. Therefore, since $M_{t}=M_{\widetilde{\xi(t)}}$, the mean curvature flow $M_{t}$ collapses to the focal submanifold $F:=M_{\widetilde{w}_{1}}$ in time $T$, where $\widetilde{w}_{1}$ is the parallel normal vector field of $M$ with $\left(\widetilde{w}_{1}\right)_{x_{0}}=w_{1}$. Also, $M_{t}$ 's $(-S<t<T)$ are all of parallel submanifolds of $M$ collapsing to $F$ along the mean curvature flow, where 
$-S$ is the infimum of the domain of $\xi$. Thus the first-half part of the statement (i) and the statement (ii) are shown. Next we shall show the second-half part of the statement (i). Assume that $M$ is irreducible and the codimension of $M$ is greater than one and that the fibration of $M$ onto $F$ is spherical. Set $\widetilde{M}:=(\pi \circ \phi)^{-1}(M)$ and $\widetilde{F}:=(\pi \circ \phi)^{-1}(F)$. Since the fibration of $M$ onto $F$ is spherical, $\widetilde{F}$ passes through a highest dimensional stratum $\widetilde{\sigma}$ of $\partial \widetilde{C}$. Let $a_{0}$ be the element of $\{1, \cdots, \bar{r}\}$ with $\widetilde{\sigma} \subset\left(\lambda_{a_{0}}\right)_{u_{0}}^{-1}(1)$. Set $\widetilde{M}_{t}:=(\pi \circ \phi)^{-1}\left(M_{t}\right)(t \in[0, T))$, which is the mean curvature flow having $\widetilde{M}$ as initial data. Denote by $A^{t}$ (resp. $\widetilde{A}^{t}$ ) the shape tensor of $M_{t}$ (resp. $\left.\widetilde{M}_{t}\right)$. Then, since $\widetilde{M}_{t}$ is the parallel submanifold of $\widetilde{M}$ for $\widetilde{\xi(t)}^{L}$, we have

$$
\operatorname{Spec} \widetilde{A}_{v}^{t} \backslash\{0\}=\left\{\frac{\left(\lambda_{a j}\right)_{u_{0}}(v)}{1-\left(\lambda_{a j}\right)_{u_{0}}(\xi(t))} \mid a=1, \cdots, \bar{r}, j \in \mathbb{Z}\right\}
$$

for each $v \in T_{u_{0}+\xi(t)}^{\perp} \widetilde{M}_{t}=T_{u_{0}}^{\perp} \widetilde{M}$. Since $\lim _{t \rightarrow T-0} \xi(t) \in\left(\lambda_{a_{0}}\right)_{u_{0}}^{-1}(1)$ and $\lim _{t \rightarrow T-0} \xi(t) \notin\left(\lambda_{a}\right)_{u_{0}}^{-1}(1)\left(a \in\{1, \cdots, \bar{r}\} \backslash\left\{a_{0}\right\}\right)$, we have $\lim _{t \rightarrow T-0}\left(\lambda_{a_{0}}\right)_{u_{0}}(\xi(t))=1$ and $\lim _{t \rightarrow T-0}\left(\lambda_{a}\right)_{u_{0}}(\xi(t))<1\left(a \neq a_{0}\right)$. From these facts, $\xi^{\prime}(t)=\left(\widetilde{H^{\xi(t)}}\right)_{u_{0}+\xi(t)}$ and (3.1), we have

$$
\begin{aligned}
& \lim _{t \rightarrow T-0}\left\|\widetilde{A}_{v}^{t}\right\|_{\infty}^{2}(T-t) \\
= & \lim _{t \rightarrow T-0} \frac{\left(\lambda_{a_{0}}\right)_{u_{0}}(v)^{2}}{\left(1-\left(\lambda_{a_{0}}\right)_{u_{0}}(\xi(t))\right)^{2}}(T-t) \\
= & \frac{1}{2}\left(\lambda_{a_{0}}\right)_{u_{0}}(v)^{2} \lim _{t \rightarrow T-0} \frac{1}{\left(1-\left(\lambda_{a_{0}}\right)_{u_{0}}(\xi(t))\right)\left(\lambda_{a_{0}}\right)_{u_{0}}\left(\xi^{\prime}(t)\right)} \\
= & \frac{\left(\lambda_{a_{0}}\right)_{u_{0}}(v)^{2}}{2 m_{a_{0}}^{e}\left\|\left(\mathbf{n}_{a_{0}}\right)_{u_{0}}\right\|^{2}} .
\end{aligned}
$$

Hence we have

$$
\lim _{t \rightarrow T-0} \max _{v \in S_{u_{0}+\xi(t)}^{\perp}}\left\|\widetilde{M}_{t}^{t}\right\|_{v}^{2}(T-t)=\frac{1}{2 m_{a_{0}}^{e}} .
$$

Thus the mean curvature flow $\widetilde{M}_{t}$ has type I singularity. Set $\bar{v}_{t}:=(\pi \circ \phi)_{* u_{0}+\xi(t)}(v)$ and let $\left\{\lambda_{1}^{t}, \cdots, \lambda_{n}^{t}\right\}\left(\lambda_{1}^{t} \leq \cdots \leq \lambda_{n}^{t}\right)\left(\right.$ resp. $\left.\left\{\mu_{1}^{t}, \cdots, \mu_{n}^{t}\right\}\left(0 \leq \mu_{1}^{t} \leq \cdots \leq \mu_{n}^{t}\right)\right)$ be all the eigenvalues of $A_{\bar{v}_{t}}^{t}\left(\operatorname{resp} . R\left(\cdot, \bar{v}_{t}\right) \bar{v}_{t}\right)$, where $n:=\operatorname{dim} M$. Since $M$ is an irreducible equifocal submanifold of codimension greater than one by the assumption, it is homogeneous by the homogeneity theorem of Christ (see [Ch]) and hence it is a principal orbit of a Hermann action by the result of Heintze-Palais-Terng-Thorbergsson (see $[\mathrm{HPTT}]$ ) and the classification of hyperpolar actions by Kollross (see [Kol]). Furthermore, $M$ and its parallel submanifolds are curvature-adapted by the result of Goertsches-Thorbergsson (see [GT]). Therefore, $A_{\bar{v}_{t}}^{t}$ and $R\left(\cdot, \bar{v}_{t}\right) \bar{v}_{t}$ commute and hence we have

$$
\sum_{i=1}^{n} \sum_{j=1}^{n}\left(\operatorname{Ker}\left(A_{\bar{v}_{t}}^{t}-\lambda_{i}^{t} \mathrm{id}\right) \cap \operatorname{Ker}\left(R\left(\cdot, \bar{v}_{t}\right) \bar{v}_{t}-\mu_{j}^{t} \mathrm{id}\right)\right)=T_{(\pi \circ \phi)\left(u_{0}+\xi(t)\right)} M_{t} .
$$

Set $\bar{E}_{i j}^{t}:=\operatorname{Ker}\left(A_{\bar{v}_{t}}^{t}-\lambda_{i}^{t} \mathrm{id}\right) \cap \operatorname{Ker}\left(R\left(\cdot, \bar{v}_{t}\right) \bar{v}_{t}-\mu_{j}^{t}\right.$ id $)(i, j \in\{1, \cdots, n\})$ and $I_{t}:=$ 
$\left\{(i, j) \in\{1, \cdots, n\}^{2} \mid \bar{E}_{i j}^{t} \neq\{0\}\right\}$. For each $(i, j) \in I_{t}$, we have

$$
\operatorname{Spec}\left(\left.\widetilde{A}_{v}^{t}\right|_{(\pi \circ \phi)_{*}^{-1}\left(\bar{E}_{i j}^{t}\right)}\right)=\left\{\begin{array}{ll}
\left\{\frac{\sqrt{\mu_{j}^{t}}}{\arctan \frac{\sqrt{\mu_{j}^{t}}}{\lambda_{i}^{t}}+k \pi} \mid k \in \mathbb{Z}\right. \\
\left\{\lambda_{i}^{t}\right\}
\end{array}\right\} \begin{aligned}
& \left(\mu_{j}^{t} \neq 0\right) \\
& \left(\mu_{j}^{t}=0\right)
\end{aligned}
$$

in terms of Proposition 3.2 of [Koi1] and hence

$$
\left\|\widetilde{A}_{v}^{t}\right\|_{\infty}=\max \left(\left\{\frac{\sqrt{\mu_{j}^{t}}}{\arctan \frac{\sqrt{\mu_{j}^{t}}}{\left|\lambda_{i}^{t}\right|}} \mid(i, j) \in I_{t} \text { s.t. } \mu_{j}^{t} \neq 0\right\} \cup\left\{\left|\lambda_{i}^{t}\right| \mid(i, j) \in I_{t} \text { s.t. } \mu_{j}^{t}=0\right\}\right) .
$$

It is clear that $\sup _{0<t<T} \mu_{n}^{t}<\infty$. If $\lim _{t \rightarrow T-0}\left|\lambda_{i}^{t}\right|=\infty$, then we have $\lim _{t \rightarrow T-0}\left(\frac{\sqrt{\mu_{j}^{t}}}{\arctan \frac{\sqrt{\mu_{j}^{t}}}{\lambda_{i}^{t}}}\right) / \lambda_{i}^{t}=1$. Hence we have

$$
\begin{aligned}
& \lim _{t \rightarrow T-0}\left\|\widetilde{A}_{v}^{t}\right\|_{\infty}^{2}(T-t) \\
= & \max \left\{\lim _{t \rightarrow T-0}\left(\lambda_{i}^{t}\right)^{2}(T-t) \mid i=1, \cdots, n\right\} \\
= & \lim _{t \rightarrow T-0} \max \left\{\left(\lambda_{i}^{t}\right)^{2}(T-t) \mid i=1, \cdots, n\right\} \\
= & \lim _{t \rightarrow T-0}\left\|A_{\bar{v}_{t}}^{t}\right\|_{\infty}^{2}(T-t),
\end{aligned}
$$

which together with (3.2) deduces

$$
\lim _{t \rightarrow T-0}\left\|A_{\bar{v}_{t}}^{t}\right\|_{\infty}^{2}(T-t)=\frac{\left(\lambda_{a_{0}}\right)_{u_{0}}(v)^{2}}{2 m_{a_{0}}^{e}\left\|\left(\mathbf{n}_{a_{0}}\right)_{u_{0}}\right\|^{2}}
$$

Therefore we obtain

$$
\lim _{t \rightarrow T-0} \max _{v \in S_{\exp \perp(\xi(t))}^{\perp}}\left\|A_{t}^{t}\right\|_{\infty}^{2}(T-t)=\frac{1}{2 m_{a_{0}}^{e}}<\infty .
$$

Thus the mean curvature flow $M_{t}$ has type I singularity. $\square$

Next we prove Theorem B.

Proof of Theorem B. For simplicity, set $I:=\{1, \cdots, \bar{r}\}$. Let $\widetilde{\sigma}$ be a stratum of dimension greater than zero of $\partial \widetilde{C}$ and $I_{\widetilde{\sigma}}:=\left\{a \in I \mid \widetilde{\sigma} \subset\left(\lambda_{a}\right)_{u_{0}}^{-1}(1)\right\}$. Let $w_{1} \in \widetilde{\sigma}$. Denote by $F$ (resp. $\widetilde{F}$ ) the focal submanifold of $M$ (resp. $\widetilde{M}$ ) for $\widetilde{w}_{1}$ (resp. $\widetilde{w}_{1}^{L}$ ). Assume that $F$ is not minimal. Then, since Ker $\eta_{\widetilde{w}_{1} *}=\underset{a \in I_{\tilde{\sigma}}}{\oplus}\left(E_{a 0}\right)_{u_{0}}$, we have

$$
T_{u_{0}+w_{1}} \widetilde{F}=\left(\underset{a \in I \backslash I_{\tilde{\sigma}}}{\oplus} \underset{j \in \mathbf{Z}}{\oplus} \eta_{\widetilde{w}_{1} *}\left(\left(E_{a j}\right)_{u_{0}}\right)\right) \oplus\left(\underset{a \in I_{\tilde{\sigma}} j \in \mathbf{Z} \backslash\{0\}}{\oplus} \eta_{\widetilde{w}_{1} *}\left(\left(E_{a j}\right)_{u_{0}}\right)\right) .
$$

Also we have

$$
T_{u_{0}+w_{1}}^{\perp} \widetilde{F}=\left(\underset{a \in I_{\widetilde{\sigma}}}{\oplus}\left(E_{a 0}\right)_{u_{0}}\right) \oplus T_{u_{0}}^{\perp} \widetilde{M}
$$


where we identify $T_{u_{0}+w_{1}} H^{0}([0,1], \mathfrak{g})$ with $T_{u_{0}} H^{0}([0,1], \mathfrak{g})$. For $v \in T_{u_{0}}^{\perp} \widetilde{M}(\subset$ $\left.T_{u_{0}+w_{1}}^{\perp} \widetilde{F}\right)$, we have

$$
\left.\widetilde{A}_{v}^{\widetilde{w}_{1}^{L}}\right|_{\eta_{\widetilde{w}_{1 *}}\left(\left(E_{a j}\right)_{u_{0}}\right)}=\frac{\left(\lambda_{a j}\right)_{u_{0}}(v)}{1-\left(\lambda_{a j}\right)_{u_{0}}\left(w_{1}\right)} \text { id } \quad\left((a, j) \in\left(\left(I \backslash I_{\widetilde{\sigma}}\right) \times \mathbb{Z}\right) \cup\left(I_{\widetilde{\sigma}} \times(\mathbb{Z} \backslash\{0\})\right)\right) .
$$

Hence we have

$$
\begin{aligned}
& \operatorname{Tr}_{r} \widetilde{A}_{v}^{\widetilde{w}_{1}^{L}} \\
= & \sum_{a \in I \backslash I_{\tilde{\sigma}}}\left(\sum_{j \in \mathbf{Z}} \frac{m_{a}^{e}\left(\lambda_{a, 2 j}\right)_{u_{0}}(v)}{1-\left(\lambda_{a, 2 j}\right)_{u_{0}}\left(w_{1}\right)}+\sum_{j \in \mathbf{Z}} \frac{m_{a}^{o}\left(\lambda_{a, 2 j+1}\right)_{u_{0}}(v)}{1-\left(\lambda_{a, 2 j+1}\right)_{u_{0}}\left(w_{1}\right)}\right) \\
& +\sum_{a \in I_{\widetilde{\sigma}}}\left(\sum_{j \in \mathbf{Z} \backslash\{0\}} \frac{m_{a}^{e}\left(\lambda_{a, 2 j}\right)_{u_{0}}(v)}{1-\left(\lambda_{a, 2 j}\right)_{u_{0}}\left(w_{1}\right)}+\sum_{j \in \mathbf{Z}} \frac{m_{a}^{o}\left(\lambda_{a, 2 j+1}\right)_{u_{0}}(v)}{1-\left(\lambda_{a, 2 j+1}\right)_{u_{0}}\left(w_{1}\right)}\right) \\
= & \sum_{a \in I \backslash I_{\tilde{\sigma}}}\left(m_{a}^{e} \cot \frac{\pi}{2 b_{a}}\left(1-\left(\lambda_{a}\right)_{u_{0}}\left(w_{1}\right)\right)-m_{a}^{o} \tan \frac{\pi}{2 b_{a}}\left(1-\left(\lambda_{a}\right)_{u_{0}}\left(w_{1}\right)\right)\right) \frac{\pi}{2 b_{a}}\left(\lambda_{a}\right)_{u_{0}}(v),
\end{aligned}
$$

that is, the $T_{u_{0}}^{\perp} \widetilde{M}$-component $\left(\left(\widetilde{H}^{\widetilde{w}_{1}^{L}}\right)_{u_{0}+w_{1}}\right)_{T_{u_{0}}^{\perp}} \widetilde{M}$ of $\left(\widetilde{H}^{\widetilde{w}_{1}^{L}}\right)_{u_{0}+w_{1}}$ is equal to

$$
\sum_{a \in I \backslash I_{\widetilde{\sigma}}}\left(m_{a}^{e} \cot \frac{\pi}{2 b_{a}}\left(1-\left(\lambda_{a}\right)_{u_{0}}\left(w_{1}\right)\right)-m_{a}^{o} \tan \frac{\pi}{2 b_{a}}\left(1-\left(\lambda_{a}\right)_{u_{0}}\left(w_{1}\right)\right)\right) \frac{\pi}{2 b_{a}}\left(\mathbf{n}_{a}\right)_{u_{0}} .
$$

Denote by $\Phi_{u_{0}+w_{1}}$ the normal holonomy group of $\widetilde{F}$ at $u_{0}+w_{1}$ and $L_{u_{0}}$ be the focal leaf through $u_{0}$ for $\widetilde{w}_{1}$. Since $L_{u_{0}}=\Phi_{u_{0}+w_{1}} \cdot u_{0}$, there exists $\mu \in \Phi_{u_{0}+w_{1}}$ such that $\mu\left(T_{u_{0}}^{\perp} \widetilde{M}\right)=T_{u_{1}}^{\perp} \widetilde{F}$ for any point $u_{1}$ of $L_{u_{0}}$. On the other hand, since $\widetilde{F}$ has constant principal curvatures in the sense of $[\mathrm{HOT}],\left(\widetilde{H}^{\widetilde{w}_{1}^{L}}\right)_{u_{0}+w_{1}}$ is $\Phi_{u_{0}+w_{1}}$-invariant. Hence we have $\left(\widetilde{H}^{\widetilde{w}_{1}^{L}}\right)_{u_{0}+w_{1}} \in \underset{u \in L_{u_{0}}}{\cap} T_{u}^{\perp} \widetilde{M}$, where we note that $\bigcap_{u \in L_{u_{0}}} T_{u}^{\perp} \widetilde{M}$ contains $\widetilde{\sigma}$ as an open subset. Therefore, we obtain

$$
\begin{aligned}
\left(\widetilde{H}^{\widetilde{w}_{1}^{L}}\right)_{u_{0}+w_{1}}= & \sum_{a \in I \backslash I_{\widetilde{\sigma}}}\left(m_{a}^{e} \cot \frac{\pi}{2 b_{a}}\left(1-\left(\lambda_{a}\right)_{u_{0}}\left(w_{1}\right)\right)\right. \\
& \left.-m_{a}^{o} \tan \frac{\pi}{2 b_{a}}\left(1-\left(\lambda_{a}\right)_{u_{0}}\left(w_{1}\right)\right)\right) \frac{\pi}{2 b_{a}}\left(\mathbf{n}_{a}\right)_{u_{0}} \quad(\in T \widetilde{\sigma}) .
\end{aligned}
$$

Define a tangent vector field $X^{\widetilde{\sigma}}$ on $\widetilde{\sigma}$ by $X_{w}^{\widetilde{\sigma}}:=\left(\widetilde{H}^{\widetilde{w}^{L}}\right)_{u_{0}+w}(w \in \widetilde{\sigma})$. Let $\xi$ : $(-S, T) \rightarrow \widetilde{\sigma}$ be the maximal integral curve of $X^{\widetilde{\sigma}}$ with $\xi(0)=w_{1}$. Define a function $\rho_{\widetilde{\sigma}}$ over $\widetilde{\sigma}$ by

$$
\begin{aligned}
\rho_{\widetilde{\sigma}}(w):=-\sum_{a \in I \backslash I_{\widetilde{\sigma}}} & \left(m_{a}^{e} \log \sin \frac{\pi}{2 b_{a}}\left(1-\left(\lambda_{a}\right)_{u_{0}}(w)\right)\right. \\
& \left.+m_{a}^{o} \log \cos \frac{\pi}{2 b_{a}}\left(1-\left(\lambda_{a}\right)_{u_{0}}(w)\right)\right) \quad(w \in \widetilde{\sigma}) .
\end{aligned}
$$

It follows from the definition of $X^{\widetilde{\sigma}}$ and (3.3) that $\operatorname{grad} \rho_{\widetilde{\sigma}}=X^{\widetilde{\sigma}}$. Also we can show that $\rho_{\widetilde{\sigma}}$ is downward convex and that $\rho_{\widetilde{\sigma}}(w) \rightarrow \infty$ as $w \rightarrow \partial \widetilde{\sigma}$. Hence we see that $\rho_{\widetilde{\sigma}}$ has the only minimal point. Denote by $w_{0}$ this minimal point. It is clear that $X_{w_{0}}^{\widetilde{\sigma}}=0$. Also, by imitating the proof of Theorem A, we can show that the flow of $X^{\widetilde{\sigma}}$ starting 
any point of $\widetilde{\sigma}$ other than $w_{0}$ converges to a point of $\partial \widetilde{\sigma}$ in finite time, and that, furthermore, for each point of $\partial \widetilde{\sigma}$, there exists a unique flow of $X^{\widetilde{\sigma}}$ converging to the point. Since $F$ is not minimal by the assumption, we have $X_{w_{1}}^{\widetilde{\sigma}} \neq 0$, that is, $w_{1} \neq w_{0}$. Hence we have $T<\infty$ and $\lim _{t \rightarrow T-0} \xi(t) \in \partial \widetilde{\sigma}$. Set $w_{2}:=\lim _{t \rightarrow T-0} \xi(t)$. Therefore, since $F_{t}=M_{\widetilde{\xi(t)}}$, the mean curvature flow $F_{t}$ collapses to the lower dimensional focal submanifold $F^{\prime}:=M_{\widetilde{w}_{2}}$ in time $T$, where $\widetilde{w}_{2}$ is the parallel normal vector field of $M$ with $\left(\widetilde{w}_{2}\right)_{x_{0}}=w_{2}$. Also, $F_{t}$ 's $(-S<t<T)$ are all of focal submanifolds of $M$ through $\widetilde{\sigma}$ collapsing to $F^{\prime}$ along the mean curvature flow. Thus the first-half part of the statement (i) and the statement (ii) are shown. Also, by imitating the proof of Theorem A, we can show the second-half part of the statement (i).

4. Hermann actions of cohomogeneity two. According to the homogeneity theorem for an equifocal submanifold in a symmetric space of compact type by Christ ([Ch]), equifocal submanifolds of codimension greater than one in an irreducible compact type symmetric space are homogeneous. Hence, according to the result by Heintze-Palais-Terng-Thorbergsson ([HPTT]), they occur as principal orbits of hyperpolar actions on the symmetric space. Furthermore, by using the classification of hyperpolar actions on irreducible compact type symmetric spaces by Kollross ([Kol]), we see that they occur as principal orbits of Hermann actions on the symmetric spaces. We have only to analyze the vector field $X$ defined in the previous secton to analyze the mean curvature flows having parallel submanifolds of an equifocal submanifold $M$ as initial data. Also, we have only to analyze the vector fields $X^{\tilde{\sigma}}$ 's $(\widetilde{\sigma}$ : a simplex of $\partial \widetilde{C})$ defined in the proof of Theorem B to analyze the mean curvature flows having focal submanifolds of $M$ as initial data. In this section, we shall explicitly describe the vector field $X$ defined for principal orbits of all Hermann actions of cohomogeneity two on all irreducible symmetric spaces of compact type and rank two (see Table 3). Let $G / K$ be a symmetric space of compact type and $H$ be a symmetric subgroup of $G$. Also, let $\theta$ be an involution of $G$ with $(\operatorname{Fix} \theta)_{0} \subset K \subset \operatorname{Fix} \theta$ and $\tau$ be an invloution of $G$ with $(\operatorname{Fix} \tau)_{0} \subset H \subset \operatorname{Fix} \tau$, where Fix $\theta$ (resp. Fix $\tau$ ) is the fixed point group of $\theta$ $(\operatorname{resp} . \tau)$ and $(\operatorname{Fix} \theta)_{0}\left(\operatorname{resp} .(\operatorname{Fix} \tau)_{0}\right)$ is the identity component of $\operatorname{Fix} \theta(\operatorname{resp} . \operatorname{Fix} \tau)$. In the sequel, we assume that $\tau \circ \theta=\theta \circ \tau$. Set $L:=\operatorname{Fix}(\theta \circ \tau)$. Denote by the same symbol $\theta$ (resp. $\tau$ ) the involution of the Lie algebra $\mathfrak{g}$ of $G$ induced from $\theta$ (resp. $\tau$ ). Set $\mathfrak{k}:=\operatorname{Ker}(\theta-\mathrm{id}), \mathfrak{p}:=\operatorname{Ker}(\theta+\mathrm{id}), \mathfrak{h}:=\operatorname{Ker}(\tau-\mathrm{id})$ and $\mathfrak{q}:=\operatorname{Ker}(\tau+\mathrm{id})$. The space $\mathfrak{p}$ is identified with $T_{e K}(G / K)$. From $\theta \circ \tau=\tau \circ \theta$, we have $\mathfrak{p}=\mathfrak{p} \cap \mathfrak{h}+\mathfrak{p} \cap \mathfrak{q}$. Take a maximal abelian subspace $\mathfrak{b}$ of $\mathfrak{p} \cap \mathfrak{q}$ and let $\mathfrak{p}=\mathfrak{z}_{\mathfrak{p}}(\mathfrak{b})+\sum_{\beta \in \triangle_{+}^{\prime}} \mathfrak{p}_{\beta}$ be the root space decomposition with respect to $\mathfrak{b}$, where $\mathfrak{z}_{\mathfrak{p}}(\mathfrak{b})$ is the centralizer of $\mathfrak{b}$ in $\mathfrak{p}, \triangle_{+}^{\prime}$ is the positive root system of $\triangle^{\prime}:=\left\{\beta \in \mathfrak{b}^{*} \mid \exists X(\neq 0) \in \mathfrak{p}\right.$ s.t. $\left.\operatorname{ad}(b)^{2}(X)=-\beta(b)^{2} X(\forall b \in \mathfrak{b})\right\}$ under some lexicographic ordering of $\mathfrak{b}^{*}$ and $\mathfrak{p}_{\beta}:=\left\{X \in \mathfrak{p} \mid \operatorname{ad}(b)^{2}(X)=-\beta(b)^{2} X(\forall b \in \mathfrak{b})\right\}$ $\left(\beta \in \triangle_{+}^{\prime}\right)$. Also, let $\triangle_{+}^{\prime V}:=\left\{\beta \in \triangle_{+}^{\prime} \mid \mathfrak{p}_{\beta} \cap \mathfrak{q} \neq\{0\}\right\}$ and $\triangle_{+}^{\prime H}:=\left\{\beta \in \triangle_{+}^{\prime} \mid \mathfrak{p}_{\beta} \cap \mathfrak{h} \neq\right.$ $\{0\}\}$. Then we have $\mathfrak{q}=\mathfrak{b}+\sum_{\beta \in \triangle^{\prime} V}\left(\mathfrak{p}_{\beta} \cap \mathfrak{q}\right)$ and $\mathfrak{h}=\mathfrak{z}_{\mathfrak{h}}(\mathfrak{b})+\sum_{\beta \in \triangle^{\prime H}}\left(\mathfrak{p}_{\beta} \cap \mathfrak{h}\right)$, where $\mathfrak{z} \mathfrak{h}(\mathfrak{b})$ is the centralizer of $\mathfrak{b}$ in $\mathfrak{h}$. The orbit $H(e K)$ is a reflective submanifold and it is isometric to the symmetric space $H / H \cap K$ (equipped with a metric scaled suitably). Also, $\exp ^{\perp}\left(T_{e K}^{\perp}(H(e K))\right)$ is also a reflective submanifold and it is isometric to the symmetric space $L / H \cap K$ (equipped with a metric scaled suitably), where $\exp ^{\perp}$ is the normal exponential map of $H(e K)$. The system ${\triangle^{\prime}}^{V}:={\triangle^{\prime}}_{+}^{V} \cup\left(-{\triangle^{\prime}}_{+}\right)$is the root 
system of $L / H \cap K$. Define a subset $\widetilde{C}$ of $\mathfrak{b}$ by

$$
\widetilde{C}:=\left\{b \in \mathfrak{b} \mid 0<\beta<\pi\left(\forall \beta \in{\triangle^{\prime}}_{+}^{V}\right),-\frac{\pi}{2}<\beta<\frac{\pi}{2}\left(\forall \beta \in{\triangle^{\prime}}_{+}^{H}\right)\right\} .
$$

Set $C:=\operatorname{Exp}(\widetilde{C})$, where $\operatorname{Exp}$ is the exponential map of $G / K$ at $e K$. Let $P(G, H \times$ $K):=\left\{g \in H^{1}([0,1], G) \mid(g(0), g(1)) \in H \times K\right\}$, where $H^{1}([0,1], G)$ is the Hilbert Lie group of all $H^{1}$-paths in $G$. This group acts on $H^{0}([0,1], \mathfrak{g})$ as gauge action. The orbits of the $P(G, H \times K)$-action are the inverse images of orbits of the $H$-action by $\pi \circ \phi$. The set $\Sigma:=\operatorname{Exp}(\mathfrak{b})$ is a section of the $H$-action and $\mathfrak{b}$ is a section of the $P(G, H \times K)$-action on $H^{0}([0,1], \mathfrak{g})$, where $\mathfrak{b}$ is identified with the horizontal lift of $\mathfrak{b}$ to the zero element $\hat{0}$ of $H^{0}([0,1], \mathfrak{g})(\hat{0}$ : the constant path at the zero element 0 of $\mathfrak{g})$. The set $\widetilde{C}$ is the fundamental domain of the Coxeter group of a principal $P(G, H \times K)$-orbit and each prinicipal $H$-orbit meets $C$ at one point and each singular $H$-orbit meets $\partial C$ at one point. The focal set of the principal orbit $P(G, H \times K) \cdot Z_{0}\left(Z_{0} \in \widetilde{C}\right)$ consists of the hyperplanes $\beta^{-1}(j \pi)$ 's $\left(\beta \in{\triangle^{\prime}}_{+}^{\prime V} \backslash \triangle^{\prime H}{ }_{+}, j \in\right.$ $\mathbb{Z}), \beta^{-1}\left(\left(j+\frac{1}{2}\right) \pi\right)$ 's $\left(\beta \in{\triangle^{\prime}}_{+}^{H} \backslash \triangle^{\prime}{ }_{+}, j \in \mathbb{Z}\right), \beta^{-1}\left(\frac{j \pi}{2}\right)$ 's $\left(\beta \in{\triangle^{\prime}}_{+}^{V} \cap{\triangle^{\prime}}_{+}^{H}, j \in \mathbb{Z}\right)$ in $\mathfrak{b}\left(=T_{Z_{0}}^{\perp}\left(P(G, H \times K) \cdot Z_{0}\right)\right)$. Denote by $\exp ^{G}$ the exponential map of $G$. Note that $\left.\pi \circ \exp ^{G}\right|_{\mathfrak{p}}=\operatorname{Exp}$. Let $Y_{0} \in \widetilde{C}$ and $M\left(Y_{0}\right):=H\left(\operatorname{Exp}\left(Y_{0}\right)\right)$. Then we have $T_{\operatorname{Exp}\left(Y_{0}\right)}^{\perp} M\left(Y_{0}\right)=\left(\exp ^{G}\left(Y_{0}\right)\right)_{*}(\mathfrak{b})$. Denote by $A^{Y_{0}}$ the shape tensor of $M\left(Y_{0}\right)$. Take $v \in T_{\operatorname{Exp}\left(Y_{0}\right)}^{\perp} M\left(Y_{0}\right)$ and set $\bar{v}:=\left(\exp ^{G}\left(Y_{0}\right)\right)_{*}^{-1}(v)$. By scaling the metric of $G / K$ by a suitable positive constant, we have

$$
\left.A_{v}^{Y_{0}}\right|_{\exp ^{G}\left(Y_{0}\right)_{*}\left(\mathfrak{p}_{\beta} \cap \mathfrak{q}\right)}=-\frac{\beta(\bar{v})}{\tan \beta\left(Y_{0}\right)} \mathrm{id}\left(\beta \in{\triangle^{\prime}}_{+}^{V}\right)
$$

and

$$
\left.A_{v}^{Y_{0}}\right|_{\exp ^{G}\left(Y_{0}\right)_{*}\left(\mathfrak{p}_{\beta} \cap \mathfrak{h}\right)}=\beta(\bar{v}) \tan \beta\left(Y_{0}\right) \text { id }\left(\beta \in{\triangle^{\prime}}_{+}^{H}\right) .
$$

Set $m_{\beta}^{V}:=\operatorname{dim}\left(\mathfrak{p}_{\beta} \cap \mathfrak{q}\right)\left(\beta \in{\triangle^{\prime}}_{+}^{V}\right)$ and $m_{\beta}^{H}:=\operatorname{dim}\left(\mathfrak{p}_{\beta} \cap \mathfrak{h}\right)\left(\beta \in \triangle_{\triangle_{+}^{\prime H}}^{\prime}\right)$. Set $\widetilde{M}\left(Y_{0}\right):=$ $(\pi \circ \phi)^{-1}\left(M\left(Y_{0}\right)\right)\left(=P(G, H \times K) \cdot Y_{0}\right)$. We can show $(\pi \circ \phi)\left(Y_{0}\right)=\operatorname{Exp}\left(Y_{0}\right)$. Denote by $\widetilde{A}^{Y_{0}}$ the shape tensor of $\widetilde{M}\left(Y_{0}\right)$. According to Proposition 3.2 of [Koi1], we have

$$
\begin{aligned}
& \operatorname{Spec}\left(\left.\widetilde{A}_{\bar{v}}^{Y_{0}}\right|_{(\pi \circ \phi)_{* Y_{0}}^{-1}\left(\exp ^{G}\left(Y_{0}\right)_{*}\left(\mathfrak{p}_{\beta} \cap \mathfrak{q}\right)\right)}\right) \backslash\{0\}=\left\{\frac{-\beta(\bar{v})}{\beta\left(Y_{0}\right)+j \pi} \mid j \in \mathbb{Z}\right\} \quad\left(\beta \in{\triangle^{\prime}}_{+}^{V}\right), \\
& \operatorname{Spec}\left(\left.\widetilde{A}_{\bar{v}}^{Y_{0}}\right|_{(\pi \circ \phi)_{* Y_{0}}^{-1}\left(\exp ^{G}\left(Y_{0}\right)_{*}\left(\mathfrak{p}_{\beta} \cap \mathfrak{h}\right)\right)}\right) \backslash\{0\}=\left\{\frac{-\beta(\bar{v})}{\beta\left(Y_{0}\right)+\left(j+\frac{1}{2}\right) \pi} \mid j \in \mathbb{Z}\right\} \quad\left(\beta \in{\triangle^{\prime}}_{+}^{H}\right),
\end{aligned}
$$

and

$$
\operatorname{Spec}\left(\left.\widetilde{A}_{\bar{v}}^{Y_{0}}\right|_{(\pi \circ \phi)_{* Y_{0}}^{-1}\left(\exp ^{G}\left(Y_{0}\right)_{*}(\mathfrak{z} \mathfrak{h}(\mathfrak{b}))\right)}\right)=\{0\}
$$

Hence the set $\mathcal{P} \mathcal{C}_{\widetilde{M}\left(Y_{0}\right)}$ of all principal curvatures of $\widetilde{M}\left(Y_{0}\right)$ is given by

$$
\mathcal{P C}_{\widetilde{M}\left(Y_{0}\right)}=\left\{\frac{-\widetilde{\beta}}{\beta\left(Y_{0}\right)+j \pi} \mid \beta \in{\triangle^{\prime}}_{+}^{V}, j \in \mathbb{Z}\right\} \cup\left\{\frac{-\widetilde{\beta}}{\beta\left(Y_{0}\right)+\left(j+\frac{1}{2}\right) \pi} \mid \beta \in{\triangle^{\prime}}_{+}^{H}, j \in \mathbb{Z}\right\},
$$

where $\widetilde{\beta}$ is the parallel section of $\left(T^{\perp} \widetilde{M}\left(Y_{0}\right)\right)^{*}$ with $\widetilde{\beta}_{u_{0}}=\beta \circ \exp ^{G}\left(Y_{0}\right)_{*}^{-1}$. Also, we can show that the multiplicity of $\frac{-\widetilde{\beta}}{\beta\left(Y_{0}\right)+j \pi}\left(\beta \in{\triangle^{\prime}}_{+}^{V}\right)$ is equal to $m_{\beta}^{V}$ and that of 
$\frac{-\widetilde{\beta}}{\beta\left(Y_{0}\right)+\left(j+\frac{1}{2}\right) \pi}\left(\beta \in{\triangle^{\prime}}_{+}^{H}\right)$ is equal to $m_{\beta}^{H}$. Define $\lambda_{\beta}^{Y_{0}}$ and $b_{\beta}^{Y_{0}}\left(\beta \in{\triangle^{\prime}}_{+}\right)$by

$$
\left(\lambda_{\beta}^{Y_{0}}, b_{\beta}^{Y_{0}}\right):= \begin{cases}\left(\frac{-\widetilde{\beta}}{\beta\left(Y_{0}\right)}, \frac{\pi}{\beta\left(Y_{0}\right)}\right) & \left(\beta \in{\triangle^{\prime}}_{+}^{V} \backslash \triangle_{+}^{\prime H}\right) \\ \left(\frac{-\widetilde{\beta}}{\left.\beta\left(Y_{0}\right)+\frac{\pi}{2}, \frac{\pi}{\beta\left(Y_{0}\right)+\frac{\pi}{2}}\right)}\right. & \left(\beta \in{\triangle^{\prime}}_{+}^{H} \backslash \triangle_{+}^{\prime V}\right) \\ \left(\frac{-\widetilde{\beta}}{\beta\left(Y_{0}\right)}, \frac{\pi}{2 \beta\left(Y_{0}\right)}\right) & \left(\beta \in{\triangle^{\prime}}_{+}^{V} \cap \triangle_{+}^{\prime H}\right) .\end{cases}
$$

Then we have $\frac{-\widetilde{\beta}}{\beta\left(Y_{0}\right)+j \pi}=\frac{\lambda_{\beta}^{Y_{0}}}{1+j b_{\beta}^{Y_{0}}}$ when $\beta \in \triangle_{+}^{\prime V} \backslash \triangle_{+}^{\prime H}, \frac{-\widetilde{\beta}}{\beta\left(Y_{0}\right)+\left(j+\frac{1}{2}\right) \pi}=\frac{\lambda_{\beta}^{Y_{0}}}{1+j b_{\beta}^{Y_{0}}}$ when $\beta \in{\triangle^{\prime}}_{+}^{H} \backslash \triangle_{+}^{\prime V}$ and $\left(\frac{-\widetilde{\beta}}{\beta\left(Y_{0}\right)+j \pi}, \frac{-\widetilde{\beta}}{\beta\left(Y_{0}\right)+\left(j+\frac{1}{2}\right) \pi}\right)=\left(\frac{\lambda_{\beta}^{Y_{0}}}{1+2 j b_{\beta}^{Y_{0}}}, \frac{\lambda_{\beta}^{Y_{0}}}{1+(2 j+1) b_{\beta}^{Y_{0}}}\right)$ when $\beta \in{\triangle^{\prime}}_{+}^{V} \cap$ $\triangle_{+}^{\prime H}$. That is, we have

$$
\mathcal{P C}_{\widetilde{M}\left(Y_{0}\right)}=\left\{\frac{\lambda_{\beta}^{Y_{0}}}{1+j b_{\beta}^{Y_{0}}} \mid \beta \in \triangle_{+}^{\prime}, j \in \mathbb{Z}\right\} .
$$

Denote by $m_{\beta j}$ the multiplicity of $\frac{\lambda_{\beta}}{1+j b_{\beta}}$. Then we have

$$
m_{\beta, 2 j}=\left\{\begin{array}{ll}
m_{\beta}^{V} & \left(\beta \in \triangle_{+}^{\prime V} \backslash \triangle^{\prime H}\right) \\
m_{\beta}^{H} & \left(\beta \in \triangle_{+}^{\prime H} \backslash \triangle_{+}^{\prime V}\right) \\
m_{\beta}^{V} & \left(\beta \in \triangle_{+}^{\prime V} \cap \triangle_{+}^{\prime H}\right),
\end{array} \quad m_{\beta, 2 j+1}= \begin{cases}m_{\beta}^{V} & \left(\beta \in \triangle_{+}^{\prime V} \backslash \triangle_{+}^{\prime H}\right) \\
m_{\beta}^{H} & \left(\beta \in \triangle_{+}^{\prime H} \backslash \triangle_{+}^{\prime V}\right) \\
m_{\beta}^{H} & \left(\beta \in \triangle_{+}^{\prime V} \cap \triangle_{+}^{\prime H}\right),\end{cases}\right.
$$

where $j \in \mathbb{Z}$. Denote by $\widetilde{H}^{Y_{0}}$ the mean curvature vector of $\widetilde{M}\left(Y_{0}\right)$ and $\mathbf{n}_{\beta}^{Y_{0}}$ the curvature normal corresponding to $\lambda_{\beta}^{Y_{0}}$. Define $\beta^{\sharp}(\in \mathfrak{b})$ by $\beta(\cdot)=\left\langle\beta^{\sharp}, \cdot\right\rangle$ and let $\widetilde{\beta}^{\sharp} Y_{0}$ be the parallel normal vector field of $\widetilde{M}\left(Y_{0}\right)$ with $\left(\widetilde{\beta}^{\sharp} Y_{0}\right)_{Y_{0}}=\beta^{\sharp}$, where we identify $\mathfrak{b}$ with $T_{Y_{0}}^{\perp} \widetilde{M}\left(Y_{0}\right)$. From (3.1) (the case of $w=0$ ), we have

$$
\begin{aligned}
\widetilde{H}^{Y_{0}}= & \sum_{\beta \in \triangle^{\prime}+} m_{\beta}^{V} \cot \frac{\pi}{2 b_{\beta}^{Y_{0}}} \cdot \frac{\pi}{2 b_{\beta}^{Y_{0}}} \mathbf{n}_{\beta}^{Y_{0}} \\
& -\sum_{\beta \in \triangle^{\prime}+}^{+} m_{\beta}^{H} \tan \frac{\pi}{2 b_{\beta}^{Y_{0}}} \cdot \frac{\pi}{2 b_{\beta}^{Y_{0}}} \mathbf{n}_{\beta}^{Y_{0}} \\
= & -\sum_{\beta \in \triangle_{+}^{\prime}+} m_{\beta}^{V} \cot \beta\left(Y_{0}\right) \widetilde{\beta}^{Y^{Y_{0}}}+\sum_{\beta \in \triangle_{+}^{\prime}} m_{\beta}^{H} \tan \beta\left(Y_{0}\right) \widetilde{\beta}^{Y_{0}} .
\end{aligned}
$$

Define a tangent vector field $X$ on $\widetilde{C}$ by assigning $\left(\widetilde{H}^{Y_{0}}\right)_{Y_{0}}\left(\in T_{Y_{0}}^{\perp} \widetilde{M}\left(Y_{0}\right)=\mathfrak{b}(\subset V)\right)$ to each $Y_{0} \in \widetilde{C}$. From (4.3), we have

$$
X_{Y_{0}}=-\sum_{\beta \in \triangle_{+}^{\prime}} m_{\beta}^{V} \cot \beta\left(Y_{0}\right) \beta^{\sharp}+\sum_{\beta \in \triangle_{+}^{\prime H}+} m_{\beta}^{H} \tan \beta\left(Y_{0}\right) \beta^{\sharp} .
$$

By using this description, we can explicitly describe this vector field $X$ for all Hermann actions of cohomogeneity two on all irreducible symmetric spaces of compact type and rank two. All Hermann actions of cohomogeneity two on all irreducible symmetric spaces of compact type and rank two are given in Table 1 . The systems $\triangle_{+}^{\prime V}$ and $\triangle^{\prime H}$ 
for the Hermann actions are given in Table 2 and the explicit descriptions of $X$ for the Hermann actions are given in Table 3. In Table $1, H^{*} \curvearrowright G^{*} / K$ implies the dual action of $H \curvearrowright G / K$ and $L^{*} / H \cap K$ is the dual of $L / H \cap K$. In Table $2,\{\alpha, \beta, \alpha+\beta\}$ implies a positive root system of the root system of $\left(\mathfrak{a}_{2}\right)$-type $(\alpha=(2,0), \beta=(-1, \sqrt{3}))$, $\{\alpha, \beta, \alpha+\beta, 2 \alpha+\beta\}$ implies a positive root system of the root system of $\left(\mathfrak{b}_{2}\right)\left(=\left(\mathfrak{c}_{2}\right)\right)$ type $(\alpha=(1,0), \beta=(-1,1))$ and $\{\alpha, \beta, \alpha+\beta, \alpha+2 \beta, \alpha+3 \beta, 2 \alpha+3 \beta\}$ implies a positive root system of the root system of $\left(\mathfrak{g}_{2}\right)$-type $(\alpha=(2 \sqrt{3}, 0), \beta=(-\sqrt{3}, 1))$. In Table $1 \sim 3, \rho_{i}(i=1, \cdots, 16)$ imply automorphisms of $G$ and $(\cdot)^{2}$ implies the product Lie group $(\cdot) \times(\cdot)$ of a Lie group $(\cdot)$. In Table $2, \underset{(m)}{\alpha}$ and so on imply that the multiplicity of $\alpha$ is equal to $m$.

\begin{tabular}{|c|c|c|}
\hline$H \curvearrowright G / K$ & $H^{*} \curvearrowright G^{*} / K$ & $L^{*} / H \cap K$ \\
\hline$\rho_{1}(S O(3)) \curvearrowright S U(3) / S O(3)$ & $S O_{0}(1,2) \curvearrowright S L(3, \mathbb{R}) / S O(3)$ & $(S L(2, \mathbb{R}) / S O(2)) \times \mathbb{R}$ \\
\hline$S O(6) \curvearrowright S U(6) / S p(3)$ & $S O^{*}(6) \curvearrowright S U^{*}(6) / S p(3)$ & $S L(3, \mathbb{C}) / S U(3)$ \\
\hline$\rho_{2}(S p(3)) \curvearrowright S U(6) / S p(3)$ & $S p(1,2) \curvearrowright S U^{*}(6) / S p(3)$ & $\left(S U^{*}(4) / S p(2)\right) \times U(1)$ \\
\hline $\begin{array}{c}S O(q+2) \curvearrowright \\
S U(q+2) / S(U(2) \times U(q))\end{array}$ & $\begin{array}{c}S O_{0}(2, q) \curvearrowright \\
S U(2, q) / S(U(2) \times U(q))\end{array}$ & $S O_{0}(2, q) / S O(2) \times S O(q)$ \\
\hline $\begin{array}{c}S(U(j+1) \times U(q-j+1)) \curvearrowright \\
S U(q+2) / S(U(2) \times U(q))\end{array}$ & $\begin{array}{l}S(U(1, j) \times U(1, q-j)) \curvearrowright \\
S U(2, q) / S(U(2) \times U(q))\end{array}$ & $\begin{array}{c}(S U(1, j) / S(U(1) \times U(j))) \times \\
(S U(1, q-j) / S(U(1) \times U(q-j)))\end{array}$ \\
\hline $\begin{array}{c}S O(j+1) \times S O(q-j+1) \curvearrowright \\
S O(q+2) / S O(2) \times S O(q)\end{array}$ & $\begin{array}{l}S O(1, j) \times S O(1, q-j) \curvearrowright \\
S O(2, q) / S O(2) \times S O(q)\end{array}$ & $\begin{array}{c}\left(S O_{0}(1, j) / S O(j)\right) \times \\
\left(S O_{0}(1, q-j) / S O(q-j)\right)\end{array}$ \\
\hline $\begin{array}{c}S O(4) \times S O(4) \curvearrowright \\
S O(8) / U(4)\end{array}$ & $\begin{array}{c}S O^{*}(4) \times S O^{*}(4) \curvearrowright \\
S O^{*}(8) / U(4)\end{array}$ & $S U(2,2) / S(U(2) \times U(2))$ \\
\hline $\begin{array}{c}\rho_{3}(S O(4) \times S O(4)) \curvearrowright \\
S O(8) / U(4)\end{array}$ & $S O(4, \mathbb{C}) \curvearrowright S O^{*}(8) / U(4)$ & $S O(4, \mathbb{C}) / S O(4)$ \\
\hline$\rho_{4}(U(4)) \curvearrowright S O(8) / U(4)$ & $U(2,2) \curvearrowright S O^{*}(8) / U(4)$ & $\left(S O^{*}(4) / U(2)\right) \times\left(S O^{*}(4) / U(2)\right)$ \\
\hline $\begin{array}{c}S O(4) \times S O(6) \curvearrowright \\
S O(10) / U(5)\end{array}$ & $\begin{array}{c}S O^{*}(4) \times S O^{*}(6) \curvearrowright \\
S O^{*}(10) / U(5)\end{array}$ & $S U(2,3) / S(U(2) \times U(3))$ \\
\hline $\begin{array}{c}S O(5) \times S O(5) \curvearrowright \\
S O(10) / U(5)\end{array}$ & $\left.S O^{(} 5, \mathbb{C}\right) \curvearrowright S O^{*}(10) / U(5)$ & $S O(5, \mathbb{C}) / S O(5)$ \\
\hline$\rho_{5}(U(5)) \curvearrowright S O(10) / U(5)$ & $U(2,3) \curvearrowright S O^{*}(10) / U(5)$ & $\left(S O^{*}(4) / U(2)\right) \times\left(S O^{*}(6) / U(3)\right)$ \\
\hline $\begin{array}{c}S O(2)^{2} \times S O(3)^{2} \curvearrowright \\
(S O(5) \times S O(5)) / S O(5)\end{array}$ & $\begin{array}{c}S O(2, \mathbb{C}) \times S O(3, \mathbb{C}) \curvearrowright \\
S O(5, \mathbb{C}) / S O(5)\end{array}$ & $S O_{0}(2,3) / S O(2) \times S O(3)$ \\
\hline $\begin{array}{c}\rho_{6}(S O(5)) \curvearrowright \\
(S O(5) \times S O(5)) / S O(5)\end{array}$ & $S O_{0}(2,3) \curvearrowright S O(5, \mathbb{C}) / S O(5)$ & $\begin{aligned} &(S O(2, \mathbb{C}) / S O(2)) \\
& \times(S O(3, \mathbb{C}) / S O(3)) \\
&\end{aligned}$ \\
\hline$\rho_{7}(U(2)) \curvearrowright S p(2) / U(2)$ & $U(1,1) \curvearrowright S p(2, \mathbb{R}) / U(2)$ & $\begin{array}{l}(S p(1, \mathbb{R}) / U(1)) \\
\times(S p(1, \mathbb{R}) / U(1))\end{array}$ \\
\hline $\begin{array}{c}S U(q+2) \curvearrowright \\
S p(q+2) / S p(2) \times S p(q)\end{array}$ & $\begin{array}{c}S U(2, q) \curvearrowright \\
S p(2, q) / S p(2) \times S p(q)\end{array}$ & $S U(2, q) / S(U(2) \times U(q))$ \\
\hline
\end{tabular}

TABLE 1. 


\begin{tabular}{|c|c|c|}
\hline$H \curvearrowright G / K$ & $H^{*} \curvearrowright G^{*} / K$ & $L^{*} / H \cap K$ \\
\hline $\begin{array}{c}U(4) \curvearrowright \\
S p(4) / S p(2) \times S p(2)\end{array}$ & $\begin{array}{c}U^{*}(4) \curvearrowright \\
S p(2,2) / S p(2) \times S p(2)\end{array}$ & $S p(2, \mathbb{C}) / S p(2)$ \\
\hline $\begin{array}{c}S p(j+1) \times S p(q-j+1) \curvearrowright \\
S p(q+2) / S p(2) \times S p(q)\end{array}$ & $\begin{array}{c}S p(1, j) \times S p(1, q-j) \curvearrowright \\
S p(2, q) / S p(2) \times S p(q)\end{array}$ & $\begin{array}{c}(S p(1, j) / S p(1) \times S p(j)) \times \\
(S p(1, q-j) / S p(1) \times S p(q-j))\end{array}$ \\
\hline $\begin{array}{c}S U(2)^{2} \cdot S O(2)^{2} \curvearrowright \\
(S p(2) \times S p(2)) / S p(2)\end{array}$ & $\begin{array}{c}S L(2, \mathbb{C}) \cdot S O(2, \mathbb{C}) \curvearrowright \\
S p(2, \mathbb{C}) / S p(2)\end{array}$ & $S p(2, \mathbb{R}) / U(2)$ \\
\hline $\begin{array}{c}\rho_{8}(S p(2)) \curvearrowright \\
(S p(2) \times S p(2)) / S p(2) \\
\end{array}$ & $S p(2, \mathbb{R}) \curvearrowright S p(2, \mathbb{C}) / S p(2)$ & $\begin{aligned} &(S L(2, \mathbb{C}) / S U(2)) \\
& \times(S O(2, \mathbb{C}) / S O(2)) \\
&\end{aligned}$ \\
\hline $\begin{array}{c}\rho_{9}(S p(2)) \curvearrowright \\
(S p(2) \times S p(2)) / S p(2)\end{array}$ & $S p(1,1) \curvearrowright S p(2, \mathbb{C}) / S p(2)$ & $\begin{aligned} &(S p(1, \mathbb{C}) / S p(1)) \\
& \times(S p(1, \mathbb{C}) / S p(1))\end{aligned}$ \\
\hline$S p(4) \curvearrowright E_{6} / \operatorname{Spin}(10) \cdot U(1)$ & $S p(2,2) \curvearrowright E_{6}^{-14} / \operatorname{Spin}(10) \cdot U(1)$ & $S p(2,2) / S p(2) \times S p(2)$ \\
\hline $\begin{array}{c}S U(6) \cdot S U(2) \curvearrowright \\
E_{6} / \operatorname{Spin}(10) \cdot U(1)\end{array}$ & $\begin{array}{c}S U(2,4) \cdot S U(2) \curvearrowright \\
E_{6}^{-14} / \operatorname{Spin}(10) \cdot U(1) \\
\end{array}$ & $S U(2,4) / S(U(2) \times U(4))$ \\
\hline $\begin{array}{c}\rho_{10}(S U(6) \cdot S U(2)) \curvearrowright \\
E_{6} / \operatorname{Spin}(10) \cdot U(1)\end{array}$ & $\begin{array}{l}S U(1,5) \cdot S L(2, \mathbb{R}) \curvearrowright \\
E_{6}^{-14} / \operatorname{Spin}(10) \cdot U(1)\end{array}$ & $S O^{*}(10) / U(5)$ \\
\hline $\begin{array}{c}\rho_{11}(\operatorname{Spin}(10) \cdot U(1)) \curvearrowright \\
E_{6} / \operatorname{Spin}(10) \cdot U(1)\end{array}$ & $\begin{array}{c}S O^{*}(10) \cdot U(1) \curvearrowright \\
E_{6}^{-14} / \operatorname{Spin}(10) \cdot U(1) \\
\end{array}$ & $\begin{array}{c}(S U(1,5) / S(U(1) \times U(5))) \\
\quad \times(S L(2, \mathbb{R}) / S O(2))\end{array}$ \\
\hline $\begin{array}{c}\rho_{12}(\operatorname{Spin}(10) \cdot U(1)) \curvearrowright \\
E_{6} / \operatorname{Spin}(10) \cdot U(1)\end{array}$ & $\begin{array}{c}S O_{0}(2,8) \cdot U(1) \curvearrowright \\
E_{6}^{-14} / \operatorname{Spin}(10) \cdot U(1)\end{array}$ & $S O_{0}(2,8) / S O(2) \times S O(8)$ \\
\hline$S p(4) \curvearrowright E_{6} / F_{4}$ & $S p(1,3) \curvearrowright E_{6}^{-26} / F_{4}$ & $S U^{*}(6) / S p(3)$ \\
\hline$\rho_{13}\left(F_{4}\right) \curvearrowright E_{6} / F_{4}$ & $F_{4}^{-20} \curvearrowright E_{6}^{-26} / F_{4}$ & $\left(S O_{0}(1,9) / S O(9)\right) \times U(1)$ \\
\hline$\rho_{14}(S O(4)) \curvearrowright G_{2} / S O(4)$ & $S L(2, \mathbb{R}) \times S L(2, \mathbb{R}) \curvearrowright G_{2}^{2} / S O(4)$ & $S O(4) / S O(2) \times S O(2)$ \\
\hline$\rho_{15}(S O(4)) \curvearrowright G_{2} / S O(4)$ & $\rho_{15}^{*}(S O(4)) \curvearrowright G_{2}^{2} / S O(4)$ & $\begin{aligned} &(S L(2, \mathbb{R}) / S O(2)) \\
& \times(S L(2, \mathbb{R}) / S O(2))\end{aligned}$ \\
\hline$\rho_{16}\left(G_{2}\right) \curvearrowright\left(G_{2} \times G_{2}\right) / G_{2}$ & $G_{2}^{2} \curvearrowright G_{2}^{\mathbf{C}} / G_{2}$ & $\begin{aligned} &(S L(2, \mathbb{C}) / S U(2)) \\
& \times(S L(2, \mathbb{C}) / S U(2))\end{aligned}$ \\
\hline$S U(2)^{4} \curvearrowright\left(G_{2} \times G_{2}\right) / G_{2}$ & $S L(2, \mathbb{C}) \times S L(2, \mathbb{C}) \curvearrowright G_{2}^{\mathbf{C}} / G_{2}$ & $G_{2}^{2} / S O(4)$ \\
\hline
\end{tabular}

Table 1 (continued). 


\begin{tabular}{|c|c|c|c|}
\hline$H \curvearrowright G / K$ & $\triangle_{+}=\triangle_{+}^{\prime}$ & ${\triangle_{+}^{\prime V}}_{+}$ & $\triangle_{+}^{\prime H}$ \\
\hline$\rho_{1}(S O(3)) \curvearrowright S U(3) / S O(3)$ & $\{\underset{(1)}{\alpha, \underset{(1)}{\beta,}, \alpha+\beta}(1)$ & $\left\{\begin{array}{l}\alpha \\
(1)\end{array}\right\}$ & $\{\underset{(1)}{\beta, \alpha+\beta}, \beta\}$ \\
\hline$S O(6) \curvearrowright S U(6) / S p(3)$ & $\{\underset{(4)}{\alpha, \underset{(4)}{\beta}, \alpha+\beta\}}, \alpha+\beta\}$ & $\{\underset{(2)}{\alpha}, \underset{(2)}{\beta}, \underset{(2)}{\alpha+\beta\}}$ & $\{\underset{(2)}{\alpha}, \underset{(2)}{\beta}, \alpha \underset{(2)}{\alpha+\beta\}}$ \\
\hline$\rho_{2}(S p(3)) \curvearrowright S U(6) / S p(3)$ & $\{\underset{(4)}{\alpha}, \underset{(4)}{\beta}, \underset{(4)}{\alpha+\beta\}}$ & $\left\{\begin{array}{c}\alpha \\
(4)\end{array}\right\}$ & $\underset{(4)}{\beta}, \alpha \underset{(4)}{\beta}+\beta\}$ \\
\hline $\begin{array}{c}S O(q+2) \curvearrowright \\
S U(q+2) / S(U(2) \times U(q)) \\
(q>2)\end{array}$ & $\begin{array}{l}\left\{\begin{array}{c}\alpha \\
\alpha-4)\end{array}, \beta, \alpha+\beta,\right. \\
2 \alpha+\beta, 2 \alpha, 2 \alpha+2 \beta\} \\
2 \alpha+(2)\end{array}$ & $\begin{array}{c}\{\underset{(q-2)}{\alpha}, \underset{(1)}{\beta, \alpha+\beta} \underset{(q-2)}{\alpha+\beta} \\
2 \alpha+\beta\} \\
(1)\end{array}$ & $\begin{array}{c}\{\underset{(q-2)}{\alpha}, \underset{(1)}{\beta, \alpha+\beta}, \underset{(q-2)}{\alpha+\beta} \\
2 \alpha+\beta, \underset{(1)}{2 \alpha} \underset{(1)}{2 \alpha+2 \beta\}}\end{array}$ \\
\hline $\begin{array}{c}S(U(j+1) \times U(q-j+1)) \curvearrowright \\
S U(q+2) / S(U(2) \times U(q)) \\
(q>2)\end{array}$ & $\begin{array}{l}\{\underset{(2 q-4)}{\alpha}, \underset{(2)}{\beta, \alpha+\beta},(2 q-4) \\
2 \alpha+\beta, 2 \alpha, 2 \alpha+2 \beta\} \\
\quad(2)\end{array}$ & $\begin{array}{c}\{\underset{(2 j-2)}{\alpha}, \underset{(2 q-2 j-2)}{\alpha+\beta}, \\
2 \alpha, 2 \alpha+2 \beta\} \\
\quad(1)\end{array}$ & $\begin{array}{l}\left\{\begin{array}{c}\alpha \\
(2 q-2 j-6)\end{array}, \beta,\right. \\
\alpha+\beta, 2 \alpha+\beta\} \\
(2 j-2) \quad(2)\end{array}$ \\
\hline $\begin{array}{c}S(U(2) \times U(2)) \curvearrowright \\
S U(4) / S(U(2) \times U(2)) \\
\text { (non-isotropy gr. act.) }\end{array}$ & $\begin{array}{c}\{\underset{(2)}{\alpha} \underset{(1)}{\beta, \alpha+\beta}, \beta \\
2 \alpha+\beta\} \\
(1)\end{array}$ & $\{\underset{(1)}{\alpha}, \alpha \underset{(1)}{\alpha+\beta\}}\}$ & 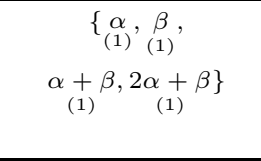 \\
\hline $\begin{array}{c}S O(j+1) \times S O(q-j+1) \curvearrowright \\
S O(q+2) / S O(2) \times S O(q)\end{array}$ & $\begin{array}{c}\{\underset{(q-2)}{\alpha}, \underset{(1)}{\beta, \alpha+\beta}(q-2) \\
2 \alpha+\beta\} \\
(1) \\
\end{array}$ & $\{\underset{(j-1)}{\alpha}, \underset{(q-j-1)}{\alpha+\beta}\}$ & $\begin{array}{c}\left\{\begin{array}{c}\alpha \\
(q-j-1)\end{array}, \begin{array}{l}\beta, \\
(1)\end{array}\right. \\
\alpha+\beta, 2 \alpha+\beta\} \\
(j-1) \quad(1)\end{array}$ \\
\hline $\begin{array}{c}S O(4) \times S O(4) \curvearrowright \\
S O(8) / U(4)\end{array}$ & $\begin{array}{c}\{\underset{(4)}{\alpha, \beta}, \beta \underset{(1)}{\beta, \alpha+\beta,} \\
2 \alpha+\beta\} \\
(1)\end{array}$ & $\begin{array}{c}\{\underset{(2)}{\alpha, \underset{(1)}{\beta, \alpha+(2)}}, \beta, \\
\left.2{ }_{(1)}+\beta\right\} \\
\end{array}$ & $\{\underset{(2)}{\alpha, \alpha+\beta\}}$ \\
\hline $\begin{array}{c}\rho_{3}(S O(4) \times S O(4)) \curvearrowright \\
S O(8) / U(4)\end{array}$ & $\begin{array}{c}\{\underset{(4)}{\alpha} \underset{(1)}{\beta, \alpha+\beta}, \beta \\
2 \alpha+\beta\} \\
(1) \\
\end{array}$ & $\{\underset{(2)}{\alpha}, \underset{(2)}{\alpha+\beta\}}$ & 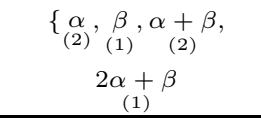 \\
\hline$\rho_{4}(U(4)) \curvearrowright S O(8) / U(4)$ & $\begin{array}{c}\{\underset{(4)}{\alpha}, \underset{(1)}{\beta, \alpha+\beta}(4) \\
2 \alpha+\beta\} \\
(1)\end{array}$ & $\{\underset{(1)}{\alpha, \alpha+\beta\}}$ & $\begin{array}{c}\{\underset{(3)}{\alpha, \underset{(1)}{\beta, \alpha+\beta}(3)} \\
2 \underset{(1)}{2 \alpha+\beta}\end{array}$ \\
\hline $\begin{array}{c}S O(4) \times S O(6) \curvearrowright \\
S O(10) / U(5)\end{array}$ & $\begin{array}{c}\left\{\begin{array}{c}\alpha \\
(4)\end{array}, \beta, \alpha+\beta,\right. \\
2 \alpha+\beta, \\
\quad(4) \\
(4)\end{array}$ & $\begin{array}{c}\{\underset{(2)}{\alpha}, \underset{(2)}{\beta}, \alpha+\beta, \\
2 \alpha+\beta \\
2 \alpha+\beta, \underset{(1)}{2 \alpha}, 2 \alpha+2 \beta\} \\
\quad(2) \\
\end{array}$ & $\begin{array}{c}\{\underset{(2)}{\alpha} \underset{(2)}{\beta, \alpha+\beta}, \beta \\
2 \alpha+\beta \\
(2) \\
\end{array}$ \\
\hline $\begin{array}{c}S O(5) \times S O(5) \curvearrowright \\
S O(10) / U(5)\end{array}$ & $\begin{array}{c}\{\underset{(4)}{\alpha}, \beta, \alpha+\beta, \\
\left.2 \alpha+\beta,{ }_{(4)} \alpha, 2 \alpha+2 \beta\right\} \\
(4) \\
(1)\end{array}$ & $\begin{array}{c}\{\underset{(2)}{\alpha, \underset{(2)}{\beta, \alpha+\beta}, \beta,} \\
2 \underset{(2)}{\alpha+\beta\}} \\
\end{array}$ & 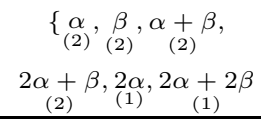 \\
\hline
\end{tabular}

TABle 2. 


\begin{tabular}{|c|c|c|c|}
\hline$H \curvearrowright G / K$ & $\Delta_{+}=\Delta_{+}^{\prime}$ & $\Delta_{+}^{\prime V}$ & $\triangle_{+}^{\prime H}$ \\
\hline$\rho_{5}(U(5)) \curvearrowright S O(10) / U(5)$ & 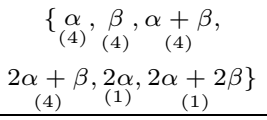 & $\{\underset{(4)}{\alpha}, \underset{(1)}{2 \alpha}, 2 \alpha+2 \beta\}$ & $\{\underset{(4)}{\beta}, \alpha+\underset{(4)}{\beta} \underset{(4)}{\beta} 2 \alpha+\beta\}$ \\
\hline $\begin{array}{c}S O(2)^{2} \times S O(3)^{2} \curvearrowright \\
(S O(5) \times S O(5)) / S O(5)\end{array}$ & $\begin{array}{c}\left\{\underset{(2)}{\alpha}, \underset{(2)}{\beta, \alpha+\beta}, \begin{array}{c}(2) \\
\{2 \alpha+\beta\} \\
(2)\end{array}\right. \\
\text { \{ }\end{array}$ & $\begin{array}{c}\{\underset{(1)}{\alpha, \beta, \beta+\beta}, \alpha+\beta \\
2 \alpha+\beta\} \\
(1)\end{array}$ & $\begin{array}{c}\left\{\begin{array}{c}\alpha, \beta, \alpha+\beta \\
(1)\end{array}\right. \\
2 \alpha+\beta\} \\
(1)\end{array}$ \\
\hline $\begin{array}{c}\rho_{6}(S O(5)) \curvearrowright \\
(S O(5) \times S O(5)) / S O(5)\end{array}$ & $\begin{array}{c}\{\underset{(2)}{\alpha, \beta}, \beta+\alpha+\beta \\
\{2 \alpha+\beta\} \\
\{2) \\
\end{array}$ & $\left\{\begin{array}{c}\alpha \\
(2)\end{array}\right\}$ & $\{\underset{(2)}{\beta}, \alpha+\underset{(2)}{\beta}, \underset{(2)}{\alpha \alpha}\}$ \\
\hline$\rho_{7}(U(2)) \curvearrowright S p(2) / U(2)$ & $\begin{array}{c}\left\{\begin{array}{c}\alpha, \beta, \beta+\beta \\
(1)\end{array} \text { (1) }\right. \\
\left\{\begin{array}{c}2 \alpha+\beta\} \\
(1)\end{array}\right. \\
\end{array}$ & $\{\underset{(1)}{\alpha}, \alpha+\beta\}$ & $\{\underset{(1)}{\beta \beta}, 2 \alpha+\beta\}$ \\
\hline & $\{\underset{(4 q-8)}{\alpha}, \underset{(4)}{\beta, \alpha+\beta}$, & $\{\underset{(2 q-4)}{\alpha}, \underset{(2)}{\beta, \alpha+\beta}\}$ & $\left\{\begin{array}{c}\alpha \\
(2 q-4)\end{array}, \beta, \alpha+\beta\right\}$ \\
\hline $\begin{array}{c}S p(q+2) / S p(2) \times S p(q) \\
(q>2) \\
\end{array}$ & $\underset{(4)}{2 \alpha+\beta, \underset{(3)}{2 \alpha}, 2 \alpha+2 \beta\}}$ & $\underset{(2)}{2 \alpha+\beta, \underset{(1)}{2 \alpha}, 2 \alpha+2 \beta\}}$ & $2 \alpha+\beta, \underset{(2)}{2 \alpha}, 2 \alpha+2 \beta\}$ \\
\hline & $\{\underset{(4)}{\alpha}, \underset{(3)}{\beta}, \alpha+\beta$ & $\{\underset{(2)}{\alpha}, \underset{(1)}{\beta}, \alpha+\beta\}$ & $\{\underset{(2)}{\alpha}, \underset{(2)}{\beta}, \alpha+\beta\}$ \\
\hline$S p(4) / S p(2) \times S p(2)$ & $\begin{array}{c}2 \alpha+\beta\} \\
(4)\end{array}$ & $\begin{array}{c}2 \alpha+\beta\} \\
(1)\end{array}$ & $\begin{array}{c}2 \alpha+\beta\} \\
(3)\end{array}$ \\
\hline$U(4) \curvearrowright$ & $\{\underset{(4)}{\alpha}, \beta, \alpha+\beta$ & $\{\underset{(2)}{\alpha}, \underset{(2)}{\beta}, \alpha+\beta\}$ & $\{\underset{(2)}{\alpha}, \underset{(1)}{\beta}, \alpha+\beta\}$ \\
\hline$S p(4) / S p(2) \times S p(2)$ & $\begin{array}{c}2 \alpha+\beta\} \\
(4)\end{array}$ & $\begin{array}{c}2 \alpha+\beta\} \\
(2)\end{array}$ & $\begin{array}{c}2 \alpha+\beta\} \\
(2)\end{array}$ \\
\hline$S p(j+1) \times S p(q-j+1) \curvearrowright$ & $\left\{\begin{array}{c}\alpha \\
\alpha(4 q-8)\end{array}, \beta, \alpha+\beta\right.$ & $\{\underset{(2 j-4)}{\alpha}, 2 \alpha, \underset{(3)}{2 \alpha+\beta}\}$ & $\left\{\begin{array}{c}\alpha \\
\alpha(4 q-4 j-4)\end{array}, \beta, \alpha+\beta, \alpha+\beta\right\}$ \\
\hline $\begin{array}{c}S p(q+2) / S p(2) \times S p(q) \\
(q>2) \\
\end{array}$ & $\underset{(4)}{2 \alpha+\beta, \underset{(3)}{2 \alpha}, 2 \alpha+2 \beta\}}$ & $2 \alpha+2 \beta\}$ & $\underset{(4)}{2 \alpha+\beta\}}$ \\
\hline $\begin{array}{c}S p(2) \times S p(2) \curvearrowright \\
S p(4) / S p(2) \times S p(2)\end{array}$ & $\begin{array}{c}\{\underset{(4)}{\alpha, \beta}, \beta, \alpha+\beta \\
2 \alpha+\beta\} \\
(4)\end{array}$ & $\{\underset{(3)}{\alpha}, \alpha+\beta\}$ & $\{\underset{(1)}{\alpha}, \underset{(3)}{\beta, 2 \alpha+\beta\}}$ \\
\hline $\begin{array}{c}S U(2)^{2} \cdot S O(2)^{2} \curvearrowright \\
(S p(2) \times S p(2)) / S p(2)\end{array}$ & $\begin{array}{c}\left\{\begin{array}{c}\alpha, \beta, \\
(2)\end{array}, \alpha+\beta\right. \\
2 \alpha+\beta\} \\
(2) \\
\end{array}$ & $\begin{array}{c}\left\{\begin{array}{c}\alpha, \beta, \beta+\beta \\
(1)\end{array}, \alpha+\beta\right. \\
2 \alpha+\beta\} \\
(1)\end{array}$ & $\begin{array}{c}\left\{\begin{array}{c}\alpha, \beta, \alpha+\beta \\
(1)\end{array}, \alpha+(1)\right. \\
2 \alpha+\beta\} \\
(1)\end{array}$ \\
\hline $\begin{array}{c}\rho_{8}(S p(2)) \curvearrowright \\
(S p(2) \times S p(2)) / S p(2)\end{array}$ & $\begin{array}{c}\{\underset{(2)}{\alpha}, \underset{(2)}{\beta, \alpha+\beta}, \beta \\
2 \alpha+\beta\} \\
(2) \\
\end{array}$ & $\{\underset{(2)}{\alpha}, \underset{(2)}{\alpha+\beta\}}$ & $\{\underset{(2)}{\beta, 2 \alpha+\beta\}}$ \\
\hline
\end{tabular}

Table 2 (Continued). 


\begin{tabular}{|c|c|c|c|}
\hline$H \curvearrowright G / K$ & $\Delta_{+}=\triangle_{+}^{\prime}$ & $\Delta_{+}^{\prime V}$ & $\Delta_{+}^{\prime H}$ \\
\hline $\begin{array}{c}\rho_{9}(S p(2)) \curvearrowright \\
(S p(2) \times S p(2)) / S p(2)\end{array}$ & $\begin{array}{c}\{\underset{(2)}{\alpha}, \beta, \alpha+\beta \\
2 \alpha+\beta\} \\
(2)\end{array}$ & $\{\underset{(2)}{\alpha}, \alpha+\beta\}$ & $\{\underset{(2)}{\beta, 2 \alpha+\beta\}}$ \\
\hline $\begin{array}{c}S p(4) \curvearrowright \\
E_{6} / \operatorname{Spin}(10) \cdot U(1)\end{array}$ & $\begin{array}{c}\{\underset{(8)}{\alpha}, \underset{(6)}{\beta, \alpha+\beta}, \alpha+\beta \\
2 \alpha+\underset{(9)}{\beta, 2 \alpha}, 2 \alpha+2 \beta\} \\
(5)\end{array}$ & $\begin{array}{c}\{\underset{(4)}{\alpha, \beta, \beta}, \alpha+\beta \\
2 \alpha+\beta\} \\
(4)\end{array}$ & $\begin{array}{c}\left\{\begin{array}{c}\alpha, \beta, \beta, \alpha+\beta \\
(4) \\
2 \alpha+\beta\} \\
(1)\end{array}\right. \\
\end{array}$ \\
\hline$S U(6) \cdot S U(2) \curvearrowright$ & $\{\underset{(8)}{\alpha}, \beta, \underset{(6)}{\beta}, \alpha+\beta$ & $\{\underset{(4)}{\alpha}, \underset{(2)}{\beta}, \underset{(4)}{\alpha+\beta}$ & $\{\underset{(4)}{\alpha}, \underset{(4)}{\beta}, \alpha+\beta$ \\
\hline$E_{6} / \operatorname{Spin}(10) \cdot U(1)$ & $\begin{array}{c}2 \alpha+\beta, \underset{(1)}{2 \alpha}, 2 \alpha+2 \beta\} \\
(1)\end{array}$ & $\begin{array}{c}2 \alpha+\beta, 2 \alpha, 2 \alpha+2 \beta\} \\
(2)\end{array}$ & $\begin{array}{c}2 \alpha+\beta\} \\
(3)\end{array}$ \\
\hline$\rho_{10}(S U(6) \cdot S U(2)) \curvearrowright$ & $\{\underset{(8)}{\alpha}, \underset{(6)}{\beta}, \underset{(9)}{\alpha+\beta}$ & $\{\underset{(4)}{\alpha}, \underset{(4)}{\beta}, \alpha+\beta$ & $\{\underset{(4)}{\alpha}, \underset{(2)}{\beta}, \alpha+\beta$ \\
\hline$E_{6} / \operatorname{Spin}(10) \cdot U(1)$ & $\underset{(5)}{2 \alpha}+\underset{(1)}{2 \alpha}, 2 \alpha+2 \beta\}$ & $\underset{(4)}{2 \alpha}+\underset{(1)}{2 \alpha}, 2 \alpha+2 \beta\}$ & $\begin{array}{r}2 \alpha+\beta\} \\
(1)\end{array}$ \\
\hline & $\{\underset{(8)}{\alpha}, \underset{(6)}{\beta}, \alpha+\beta$ & $\{\underset{(8)}{\alpha}, \underset{(1)}{2 \alpha} \underset{(1)}{2 \alpha}+2 \beta\}$ & $\{\underset{(6)}{\beta}, \alpha+\beta, \beta, 2 \alpha+\beta\}$ \\
\hline$E_{6} / \operatorname{Spin}(10) \cdot U(1)$ & $\begin{array}{c}2 \alpha+\beta, \underset{(1)}{2 \alpha}, 2 \alpha+2 \beta\} \\
(1)\end{array}$ & & \\
\hline$\rho_{12}(\operatorname{Spin}(10) \cdot U(1)) \curvearrowright$ & $\{\underset{(8)}{\alpha}, \underset{(6)}{\beta, \alpha+\beta}, \beta$ & $\{\underset{(6)}{\alpha}, \underset{(1)}{\beta}, \alpha+\beta$ & $\{\underset{(2)}{\alpha}, \beta, \alpha+\beta$ \\
\hline$E_{6} / \operatorname{Spin}(10) \cdot U(1)$ & $\underset{(5)}{2 \alpha+\beta, 2 \alpha}, 2 \alpha+2 \beta\}$ & $\begin{array}{c}2 \alpha+\beta\} \\
(1)\end{array}$ & $\begin{array}{c}2 \alpha+\beta, \underset{(1)}{2 \alpha}, 2 \alpha+2 \beta\} \\
(1)\end{array}$ \\
\hline$S p(4) \curvearrowright E_{6} / F_{4}$ & $\left\{\begin{array}{c}\alpha, \beta) \\
(8)\end{array}, \alpha+\beta\right\}$ & $\left\{\begin{array}{c}\alpha, \beta \\
(4)\end{array}, \alpha+\beta\right\}$ & $\left\{\begin{array}{c}\alpha, \beta, \beta, \alpha+\beta\} \\
(4)\end{array}\right.$ \\
\hline$\rho_{13}\left(F_{4}\right) \curvearrowright E_{6} / F_{4}$ & 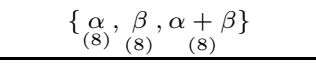 & $\left\{\begin{array}{c}\alpha \\
(8)\end{array}\right\}$ & $\{\underset{(8)}{\beta, \alpha+\beta} \underset{(8)}{\beta}\}$ \\
\hline & $\{\underset{(1)}{\alpha}, \underset{(1)}{\beta}, \alpha+\beta$ & $\{\underset{(1)}{\alpha}, 3 \alpha+2 \beta\}$ & $\{\underset{(1)}{\beta}, \alpha+\beta, \underset{(1)}{2 \alpha}+\beta$ \\
\hline$G_{2} / S O(4)$ & $\begin{array}{c}2 \alpha+\beta, 3 \alpha+\beta, 3 \alpha+2 \beta\} \\
(1) \\
\text { (1) }\end{array}$ & & $\begin{array}{c}3 \alpha+\beta\} \\
(1)\end{array}$ \\
\hline & $\{\underset{(1)}{\alpha} \underset{(1)}{\beta}, \alpha+\beta$ & $\{\underset{(1)}{\alpha}, 3 \alpha+2 \beta\}$ & $\{\underset{(1)}{\beta, \alpha+\beta} \underset{(1)}{\beta, 2 \alpha}+\beta$, \\
\hline$G_{2} / S O(4)$ & $\begin{array}{c}2 \alpha+\beta, 3 \alpha+\beta, 3 \alpha+2 \beta\} \\
(1) \\
(1)\end{array}$ & & $\begin{array}{c}3 \alpha+\beta\} \\
(1)\end{array}$ \\
\hline & $\left\{\underset{(2)}{\alpha},{ }_{(2)}^{\beta}, \alpha+\beta\right.$ & $\{\underset{(2)}{\alpha}, 3 \alpha+2 \beta\}$ & $\{\underset{(2)}{\beta, \alpha+\beta} \underset{(2)}{\beta} \underset{(2)}{\alpha \alpha}+\beta$ \\
\hline$\left(G_{2} \times G_{2}\right) / G_{2}$ & $\begin{array}{c}2 \alpha+\beta, 3 \alpha+\beta, 3 \alpha+2 \beta\} \\
(2) \\
(2)\end{array}$ & & $\begin{array}{c}3 \alpha+\beta\} \\
(2)\end{array}$ \\
\hline$S U(2)^{4} \curvearrowright$ & $\{\underset{(2)}{\alpha}, \underset{(2)}{\beta, \alpha+\beta}, \beta$ & $\{\underset{(1)}{\alpha}, \underset{(1)}{\beta}, \alpha+\beta$ & $\{\underset{(1)}{\alpha, \underset{(1)}{\beta}, \alpha+\beta}$ \\
\hline$\left(G_{2} \times G_{2}\right) / G_{2}$ & $\begin{array}{c}2 \alpha+\beta, 3 \alpha+\beta, 3 \alpha+2 \beta\} \\
(2)\end{array}$ & $\begin{array}{c}2 \alpha+\beta, 3 \alpha+\beta, 3 \alpha+2 \beta\} \\
(1)\end{array}$ & $\begin{array}{c}2 \alpha+\beta, 3 \alpha+\beta, 3 \alpha+2 \beta\} \\
(1) \\
(1)\end{array}$ \\
\hline
\end{tabular}

TABle 2 (CONTinued $\left.{ }^{2}\right)$. 


\begin{tabular}{|c|c|}
\hline$H \curvearrowright G / K$ & $(\widetilde{C})$ \\
\hline$\rho_{1}(S O(3)) \curvearrowright S U(3) / S O(3)$ & $\begin{array}{c}X_{\left(x_{1}, x_{2}\right)}=\left(\tan \left(x_{1}+\sqrt{3} x_{2}\right)-2 \cot 2 x_{1}+\tan \left(x_{1}-\sqrt{3} x_{2}\right),\right. \\
\left.\sqrt{3} \tan \left(x_{1}+\sqrt{3} x_{2}\right)-\sqrt{3} \tan \left(x_{1}-\sqrt{3} x_{2}\right)\right) \\
\left(\widetilde{C}: x_{1}>0, x_{2}>\frac{1}{\sqrt{3}} x_{1}-\frac{\pi}{2 \sqrt{3}}, x_{2}<-\frac{1}{\sqrt{3}} x_{1}+\frac{\pi}{2 \sqrt{3}}\right)\end{array}$ \\
\hline$S O(6) \curvearrowright S U(6) / S p(3)$ & $\begin{array}{c}X_{\left(x_{1}, x_{2}\right)}=\left(-4 \cot 2 x_{1}-2 \cot \left(x_{1}-\sqrt{3} x_{2}\right)-2 \cot \left(x_{1}+\sqrt{3} x_{2}\right)\right. \\
+4 \tan 2 x_{1}+2 \tan \left(x_{1}-\sqrt{3} x_{2}\right)+2 \tan \left(x_{1}+\sqrt{3} x_{2}\right) \\
2 \sqrt{3} \cot \left(x_{1}-\sqrt{3} x_{2}\right)-2 \sqrt{3} \cot \left(x_{1}+\sqrt{3} x_{2}\right) \\
\left.-2 \sqrt{3} \tan \left(x_{1}-\sqrt{3} x_{2}\right)+2 \sqrt{3} \tan \left(x_{1}+\sqrt{3} x_{2}\right)\right) \\
\left(\widetilde{C}: x_{1}>0, x_{2}>\frac{1}{\sqrt{3}} x_{1}, x_{2}<-\frac{1}{\sqrt{3}} x_{1}+\frac{\pi}{2 \sqrt{3}}\right) \\
\end{array}$ \\
\hline$\rho_{2}(S p(3)) \curvearrowright S U(6) / S p(3)$ & $\begin{array}{c}X_{\left(x_{1}, x_{2}\right)}=\left(-8 \cot 2 x_{1}+4 \tan \left(x_{1}-\sqrt{3} x_{2}\right)+4 \cot \left(x_{1}+\sqrt{3} x_{2}\right),\right. \\
\left.4 \sqrt{3} \tan \left(x_{1}+\sqrt{3} x_{2}\right)-4 \sqrt{3} \tan \left(x_{1}-\sqrt{3} x_{2}\right)\right) \\
\left(\widetilde{C}: x_{1}>0, x_{2}>\frac{1}{\sqrt{3}} x_{1}-\frac{\pi}{2 \sqrt{3}}, x_{2}<-\frac{1}{\sqrt{3}} x_{1}+\frac{\pi}{2 \sqrt{3}}\right)\end{array}$ \\
\hline $\begin{array}{c}S O(q+2) \curvearrowright \\
S U(q+2) / S(U(2) \times U(q)) \\
(q>2)\end{array}$ & $\begin{array}{c}X_{\left(x_{1}, x_{2}\right)}=\left(-(q-2) \cot x_{1}+\cot \left(x_{1}-x_{2}\right)-\cot \left(x_{1}+x_{2}\right)\right. \\
+(q-2) \tan x_{1}+\tan \left(x_{1}-x_{2}\right)+\tan \left(x_{1}+x_{2}\right)+2 \tan 2 x_{1}, \\
\cot \left(x_{1}-x_{2}\right)-(q-2) \cot x_{2}-\cot \left(x_{1}+x_{2}\right) \\
\left.-\tan \left(x_{1}-x_{2}\right)+(q-2) \tan x_{2}+\tan \left(x_{1}+x_{2}\right)+2 \tan 2 x_{2}\right) \\
\left(\widetilde{C}: x_{1}>0, x_{2}>x_{1}, x_{2}<\frac{\pi}{4}\right) \\
\end{array}$ \\
\hline $\begin{array}{c}S O(4) \curvearrowright \\
S U(4) / S(U(2) \times U(2))\end{array}$ & $\begin{array}{c}X_{\left(x_{1}, x_{2}\right)}=\left(-\cot x_{1}+\tan x_{1}+\tan \left(x_{1}-x_{2}\right)+\tan \left(x_{1}+x_{2}\right)\right. \\
\left.-\cot x_{2}-\tan \left(x_{1}-x_{2}\right)+\tan x_{2}+\tan \left(x_{1}+x_{2}\right)\right) \\
\left(\widetilde{C}: x_{1}>0, x_{2}>0, x_{1}+x_{2}<\frac{\pi}{2}\right)\end{array}$ \\
\hline $\begin{array}{c}S(U(j+1) \times U(q-j+1)) \curvearrowright \\
S U(q+2) / S(U(2) \times U(q)) \\
(q>2)\end{array}$ & $\begin{array}{c}X_{\left(x_{1}, x_{2}\right)}=\left(-2(j-1) \cot x_{1}-2 \cot 2 x_{1}+2(q-j-1) \tan x_{1}\right. \\
+2 \tan \left(x_{1}-x_{2}\right)+2 \tan \left(x_{1}+x_{2}\right) \\
-2(q-j-1) \cot x_{2}-2 \cot 2 x_{2}-2 \tan \left(x_{1}-x_{2}\right) \\
\left.+2(j-1) \tan x_{2}+2 \tan \left(x_{1}+x_{2}\right)\right) \\
\left(\widetilde{C}: x_{1}>0, x_{2}>0, x_{1}+x_{2}<\frac{\pi}{2}\right) \\
\end{array}$ \\
\hline $\begin{array}{c}S(U(2) \times U(2)) \curvearrowright \\
S U(4) / S(U(2) \times U(2)) \\
\text { (non-isotropy gr. act.) } \\
\end{array}$ & $\begin{array}{c}X_{\left(x_{1}, x_{2}\right)}=\left(-\cot x_{1}+\tan x_{1}+\tan \left(x_{1}-x_{2}\right)+\tan \left(x_{1}+x_{2}\right),\right. \\
\left.-\cot x_{2}-\tan \left(x_{1}-x_{2}\right)+\tan x_{2}+\tan \left(x_{1}+x_{2}\right)\right) \\
\left(\widetilde{C}: x_{1}>0, x_{2}>0, x_{1}+x_{2}<\frac{\pi}{2}\right)\end{array}$ \\
\hline $\begin{array}{c}S O(j+1) \times S O(q-j+1) \curvearrowright \\
S O(q+2) / S O(2) \times S O(q) \\
(q>2)\end{array}$ & $\begin{array}{c}X_{\left(x_{1}, x_{2}\right)}=\left(-(j-1) \cot x_{1}+(q-j-1) \tan x_{1}\right. \\
+\tan \left(x_{1}-x_{2}\right)+\tan \left(x_{1}+x_{2}\right) \\
-(q-j-1) \cot x_{2}-\tan \left(x_{1}-x_{2}\right) \\
\left.\quad+(j-1) \tan x_{2}+\tan \left(x_{1}+x_{2}\right)\right) \\
\left(\widetilde{C}: x_{1}>0, x_{2}>0, x_{1}+x_{2}<\frac{\pi}{2}\right)\end{array}$ \\
\hline
\end{tabular}

TABLE 3. 


\begin{tabular}{|c|c|}
\hline$H \curvearrowright G / K$ & $(\widetilde{C})$ \\
\hline $\begin{array}{c}S O(4) \times S O(4) \curvearrowright \\
S O(8) / U(4)\end{array}$ & $\begin{array}{c}X_{\left(x_{1}, x_{2}\right)}=\left(-2 \cot x_{1}-\cot \left(x_{1}-x_{2}\right)\right. \\
-2 \cot \left(x_{1}+x_{2}\right)+2 \tan x_{1} \\
\cot \left(x_{1}-x_{2}\right)-2 \cot x_{2} \\
\left.-2 \cot \left(x_{1}+x_{2}\right)+2 \tan x_{2}\right) \\
\left(\widetilde{C}: x_{1}>0, x_{2}>0, x_{2}<x_{1}, x_{1}+x_{2}<\pi\right)\end{array}$ \\
\hline $\begin{array}{c}\rho_{3}(S O(4) \times S O(4)) \curvearrowright \\
S O(8) / U(4)\end{array}$ & $\begin{array}{c}X_{\left(x_{1}, x_{2}\right)}=\left(-2 \cot x_{1}+2 \tan x_{1}\right. \\
+\tan \left(x_{1}-x_{2}\right)+\tan \left(x_{1}+x_{2}\right) \\
\quad-2 \cot x_{2}-\tan \left(x_{1}-x_{2}\right) \\
\left.\quad+2 \tan x_{2}+\tan \left(x_{1}+x_{2}\right)\right) \\
\left(\widetilde{C}: x_{1}>0, x_{2}<0, x_{1}+x_{2}<\frac{\pi}{2}\right)\end{array}$ \\
\hline$\rho_{4}(U(4)) \curvearrowright S O(8) / U(4)$ & $\begin{array}{c}X_{\left(x_{1}, x_{2}\right)}=\left(-\cot x_{1}+3 \tan x_{1}\right. \\
+\tan \left(x_{1}-x_{2}\right)+\tan \left(x_{1}+x_{2}\right) \\
-\cot x_{2}-\tan \left(x_{1}-x_{2}\right) \\
\left.+3 \tan x_{2}+\tan \left(x_{1}+x_{2}\right)\right) \\
\left(\widetilde{C}: x_{1}>0, x_{2}>0, x_{1}+x_{2}<\frac{\pi}{2}\right)\end{array}$ \\
\hline $\begin{array}{c}S O(4) \times S O(6) \curvearrowright \\
S O(10) / U(5)\end{array}$ & $\begin{array}{c}X_{\left(x_{1}, x_{2}\right)}=2\left(-\cot x_{1}-\cot \left(x_{1}-x_{2}\right)-\cot \left(x_{1}+x_{2}\right)\right. \\
-\cot 2 x_{1}+\tan x_{1} \\
+\tan \left(x_{1}-x_{2}\right)+\tan \left(x_{1}+x_{2}\right), \\
\cot \left(x_{1}-x_{2}\right)-\cot x_{2}-\cot \left(x_{1}+x_{2}\right) \\
-\cot 2 x_{2}-\tan \left(x_{1}-x_{2}\right) \\
\left.+\tan x_{2}+\tan \left(x_{1}+x_{2}\right)\right) \\
\left(\widetilde{C}: x_{1}>0, x_{2}>x_{1}, x_{1}+x_{2}<\frac{\pi}{2}\right) \\
\end{array}$ \\
\hline $\begin{array}{c}S O(5) \times S O(5) \curvearrowright \\
S O(10) / U(5)\end{array}$ & $\begin{array}{c}X_{\left(x_{1}, x_{2}\right)}=2\left(-\cot x_{1}-\cot \left(x_{1}-x_{2}\right)-\cot \left(x_{1}+x_{2}\right)\right. \\
+\tan x_{1}+\tan \left(x_{1}-x_{2}\right) \\
+\tan \left(x_{1}+x_{2}\right)+\tan 2 x_{1}, \\
\cot \left(x_{1}-x_{2}\right)-\cot x_{2}-\cot \left(x_{1}+x_{2}\right) \\
-\tan \left(x_{1}-x_{2}\right)+\tan x_{2} \\
\left.+\tan \left(x_{1}+x_{2}\right)+\tan 2 x_{2}\right) \\
\left(\widetilde{C}: x_{1}>0, x_{2}>0, x_{2}>x_{1}, x_{2}<\frac{\pi}{4}\right)\end{array}$ \\
\hline$\rho_{5}(U(5)) \curvearrowright S O(10) / U(5)$ & $\begin{array}{l}X_{\left(x_{1}, x_{2}\right)}=2\left(-2 \cot x_{1}-\cot 2 x_{1}\right. \\
+2 \tan \left(x_{1}-x_{2}\right)+2 \tan \left(x_{1}+x_{2}\right) \\
\quad-\cot 2 x_{2}-2 \tan \left(x_{1}-x_{2}\right) \\
\left.\quad+2 \tan \left(x_{1}+x_{2}\right)+2 \tan x_{2}\right) \\
\left(\widetilde{C}: x_{1}>0, x_{2}>0, x_{1}+x_{2}<\frac{\pi}{2}\right)\end{array}$ \\
\hline
\end{tabular}

Table 3 (Continued). 


\begin{tabular}{|c|c|}
\hline$H \curvearrowright G / K$ & $(\widetilde{C})$ \\
\hline $\begin{array}{c}S O(2)^{2} \times S O(3)^{2} \curvearrowright \\
(S O(5) \times S O(5)) / S O(5)\end{array}$ & $\begin{array}{c}X_{\left(x_{1}, x_{2}\right)}=\left(-\cot x_{1}-\cot \left(x_{1}-x_{2}\right)-\cot \left(x_{1}+x_{2}\right)\right. \\
+\tan x_{1}+\tan \left(x_{1}-x_{2}\right)+\tan \left(x_{1}+x_{2}\right) \\
\cot \left(x_{1}-x_{2}\right)-\cot x_{2}-\cot \left(x_{1}+x_{2}\right) \\
\left.-\tan \left(x_{1}-x_{2}\right)+\tan x_{2}+\tan \left(x_{1}+x_{2}\right)\right) \\
\left(\widetilde{C}: x_{1}>0, x_{2}>0, x_{2}>x_{1}, x_{1}+x_{2}<\frac{\pi}{2}\right)\end{array}$ \\
\hline $\begin{array}{c}\rho_{6}(S O(5)) \\
(S O(5) \times S O(5)) / S O(5)\end{array}$ & $\begin{array}{c}X_{\left(x_{1}, x_{2}\right)}=2\left(-\cot x_{1}+\tan \left(x_{1}-x_{2}\right)+\tan \left(x_{1}+x_{2}\right)\right. \\
\left.-\tan \left(x_{1}-x_{2}\right)+\tan x_{2}+\tan \left(x_{1}+x_{2}\right)\right) \\
\left(\widetilde{C}: x_{1}>0, x_{2}>x_{1}-\frac{\pi}{2}, x_{1}+x_{2}<\frac{\pi}{2}\right)\end{array}$ \\
\hline$\rho_{7}(U(2)) \curvearrowright S p(2) / U(2)$ & $\begin{array}{c}X_{\left(x_{1}, x_{2}\right)}=\left(-\cot x_{1}+\tan \left(x_{1}-x_{2}\right)+\tan \left(x_{1}+x_{2}\right)\right. \\
\left.-\cot x_{2}-\tan \left(x_{1}-x_{2}\right)+\tan \left(x_{1}+x_{2}\right)\right) \\
\left(\widetilde{C}: x_{1}>0, x_{2}>0, x_{1}+x_{2}<\frac{\pi}{2}\right)\end{array}$ \\
\hline $\begin{array}{c}U(q+2) \curvearrowright \\
S p(q+2) / S p(2) \times S p(q) \\
(q>2)\end{array}$ & $\begin{array}{c}X_{\left(x_{1}, x_{2}\right)}=\left(-(2 q-4) \cot x_{1}-2 \cot \left(x_{1}-x_{2}\right)-2 \cot \left(x_{1}+x_{2}\right)\right. \\
-2 \cot 2 x_{1}+(2 q-4) \tan x_{1}+2 \tan \left(x_{1}-x_{2}\right) \\
+2 \tan \left(x_{1}+x_{2}\right)+4 \tan 2 x_{1} \\
2 \cot \left(x_{1}-x_{2}\right)-(2 q-4) \cot x_{2}-2 \cot \left(x_{1}+x_{2}\right) \\
-2 \cot 2 x_{2}-2 \tan \left(x_{1}-x_{2}\right)+(2 q-4) \tan x_{2} \\
\left.+2 \tan \left(x_{1}+x_{2}\right)+4 \tan 2 x_{2}\right) \\
\left(\widetilde{C}: x_{1}>0, x_{2}>x_{1}, x_{2}<\frac{\pi}{4}\right)\end{array}$ \\
\hline $\begin{aligned} S U(4) & \curvearrowright \\
S p(4) / S p(2) & \times S p(2)\end{aligned}$ & $\begin{array}{c}X_{\left(x_{1}, x_{2}\right)}=\left(-2 \cot x_{1}-\cot \left(x_{1}-x_{2}\right)-\cot \left(x_{1}+x_{2}\right)\right. \\
2 \tan x_{1}+2 \tan \left(x_{1}-x_{2}\right)+3 \tan \left(x_{1}+x_{2}\right) \\
\cot \left(x_{1}-x_{2}\right)-2 \cot x_{2}-\cot \left(x_{1}+x_{2}\right) \\
\left.-2 \tan \left(x_{1}-x_{2}\right)+\tan x_{2}+3 \tan \left(x_{1}+x_{2}\right)\right) \\
\left(\widetilde{C}: x_{1}>0, x_{2}>x_{1}, x_{1}+x_{2}<\frac{\pi}{2}\right) \\
\end{array}$ \\
\hline $\begin{array}{c}U(4) \curvearrowright \\
S p(4) / S p(2) \times S p(2)\end{array}$ & $\begin{array}{c}X_{\left(x_{1}, x_{2}\right)}=\left(-2 \cot x_{1}-2 \cot \left(x_{1}-x_{2}\right)-2 \cot \left(x_{1}+x_{2}\right)\right. \\
2 \tan x_{1}+\tan \left(x_{1}-x_{2}\right)+2 \tan \left(x_{1}+x_{2}\right) \\
2 \cot \left(x_{1}-x_{2}\right)-2 \cot x_{2}-2 \cot \left(x_{1}+x_{2}\right) \\
\left.-\tan \left(x_{1}-x_{2}\right)+\tan x_{2}+2 \tan \left(x_{1}+x_{2}\right)\right) \\
\left(\widetilde{C}: x_{1}>0, x_{2}>x_{1}, x_{1}+x_{2}<\frac{\pi}{2}\right) \\
\end{array}$ \\
\hline $\begin{array}{c}S p(j+1) \times S p(q-j+1) \curvearrowright \\
S p(q+2) / S p(2) \times S p(q) \\
(q>2)\end{array}$ & $\begin{array}{c}X_{\left(x_{1}, x_{2}\right)}=\left(-4(j-1) \cot x_{1}-6 \cot 2 x_{1}\right. \\
+4(q-j-1) \tan x_{1}+4 \tan \left(x_{1}-x_{2}\right)+4 \tan \left(x_{1}+x_{2}\right) \\
-4(q-j-1) \cot x_{2}-6 \cot 2 x_{2}-4 \tan \left(x_{1}-x_{2}\right) \\
\left.+4(j-1) \tan x_{2}+4 \tan \left(x_{1}+x_{2}\right)\right) \\
\left(\widetilde{C}: x_{1}>0, x_{2}>0, x_{1}+x_{2}<\frac{\pi}{2}\right)\end{array}$ \\
\hline
\end{tabular}

Table 3 (CONTinued ${ }^{2}$ ). 


\begin{tabular}{|c|c|}
\hline$H \curvearrowright G / K$ & $(\widetilde{C})$ \\
\hline $\begin{array}{c}S p(2) \times S p(2) \curvearrowright \\
S p(4) / S p(2) \times S p(2)\end{array}$ & $\begin{array}{c}X_{\left(x_{1}, x_{2}\right)}=\left(-3 \cot x_{1}+\tan x_{1}\right. \\
+3 \tan \left(x_{1}-x_{2}\right)+4 \tan \left(x_{1}+x_{2}\right) \\
\left.-3 \cot x_{2}-3 \tan \left(x_{1}-x_{2}\right)+4 \tan \left(x_{1}+x_{2}\right)\right) \\
\left(\widetilde{C}: x_{1}>0, x_{2}>0, x_{1}+x_{2}<\frac{\pi}{2}\right)\end{array}$ \\
\hline $\begin{array}{c}S U(2)^{2} \cdot S O(2)^{2} \curvearrowright \\
(S p(2) \times S p(2)) / S p(2)\end{array}$ & $\begin{array}{c}X_{\left(x_{1}, x_{2}\right)}=\left(-\cot x_{1}-\cot \left(x_{1}-x_{2}\right)-\cot \left(x_{1}+x_{2}\right)\right. \\
+\tan x_{1}+\tan \left(x_{1}-x_{2}\right)+\tan \left(x_{1}+x_{2}\right) \\
\cot \left(x_{1}-x_{2}\right)-\cot x_{2}-\cot \left(x_{1}+x_{2}\right) \\
\left.-\tan \left(x_{1}-x_{2}\right)+\tan x_{2}+\tan \left(x_{1}+x_{2}\right)\right) \\
\left(\widetilde{C}: x_{1}>0, x_{2}>x_{1}, x_{1}+x_{2}<\frac{\pi}{2}\right) \\
\end{array}$ \\
\hline $\begin{array}{c}\rho_{8}(S p(2)) \\
(S p(2) \times S p(2)) / S p(2)\end{array}$ & $\begin{array}{c}X_{\left(x_{1}, x_{2}\right)}=2\left(-\cot x_{1}+\tan \left(x_{1}-x_{2}\right)+\tan \left(x_{1}+x_{2}\right)\right. \\
\left.-\cot x_{2}-\tan \left(x_{1}-x_{2}\right)+\tan \left(x_{1}+x_{2}\right)\right) \\
\left(\widetilde{C}: x_{1}>0, x_{2}>0, x_{1}+x_{2}<\frac{\pi}{2}\right) \\
\end{array}$ \\
\hline $\begin{array}{c}\rho_{9}(S p(2)) \curvearrowright \\
(S p(2) \times S p(2)) / S p(2)\end{array}$ & $\begin{array}{c}X_{\left(x_{1}, x_{2}\right)}=2\left(-\cot x_{1}+\tan \left(x_{1}-x_{2}\right)+\tan \left(x_{1}+x_{2}\right)\right. \\
\left.-\cot x_{2}-\tan \left(x_{1}-x_{2}\right)+\tan \left(x_{1}+x_{2}\right)\right) \\
\left(\widetilde{C}: x_{1}>0, x_{2}>0, x_{1}+x_{2}<\frac{\pi}{2}\right) \\
\end{array}$ \\
\hline $\begin{array}{c}S p(4) \curvearrowright \\
E_{6} / S p i n(10) \cdot U(1)\end{array}$ & $\begin{array}{c}X_{\left(x_{1}, x_{2}\right)}=\left(-4 \cot x_{1}-3 \cot \left(x_{1}-x_{2}\right)-4 \cot \left(x_{1}+x_{2}\right)\right. \\
+4 \tan x_{1}+3 \tan \left(x_{1}-x_{2}\right)+\tan \left(x_{1}+x_{2}\right), \\
3 \cot \left(x_{1}-x_{2}\right)-3 \cot x_{2}-4 \cot \left(x_{1}+x_{2}\right) \\
\left.-3 \tan \left(x_{1}-x_{2}\right)+6 \tan x_{2}+\tan \left(x_{1}+x_{2}\right)\right) \\
\left(\widetilde{C}: x_{1}>0, x_{2}>x_{1}, x_{1}+x_{2}<\frac{\pi}{2}\right) \\
\end{array}$ \\
\hline $\begin{array}{c}S U(6) \cdot S U(2) \curvearrowright \\
E_{6} / S \operatorname{pin}(10) \cdot U(1)\end{array}$ & $\begin{array}{c}X_{\left(x_{1}, x_{2}\right)}=\left(-4 \cot x_{1}-2 \cot \left(x_{1}-x_{2}\right)-2 \cot \left(x_{1}+x_{2}\right)\right. \\
-2 \cot 2 x_{1}+4 \tan x_{1} \\
+4 \tan \left(x_{1}-x_{2}\right)+3 \tan \left(x_{1}+x_{2}\right), \\
2 \cot \left(x_{1}-x_{2}\right)-4 \cot x_{2}-2 \cot \left(x_{1}+x_{2}\right) \\
-2 \cot 2 x_{2}-4 \tan \left(x_{1}-x_{2}\right) \\
\left.+5 \tan x_{2}+3 \tan \left(x_{1}+x_{2}\right)\right) \\
\left(\widetilde{C}: x_{1}>0, x_{2}>x_{1}, x_{1}+x_{2}<\frac{\pi}{2}\right) \\
\end{array}$ \\
\hline $\begin{array}{c}\rho_{10}(S U(6) \cdot S U(2)) \curvearrowright \\
E_{6} / \operatorname{Spin}(10) \cdot U(1)\end{array}$ & $\begin{array}{c}X_{\left(x_{1}, x_{2}\right)}=\left(-4 \cot x_{1}-4 \cot \left(x_{1}-x_{2}\right)-4 \cot \left(x_{1}+x_{2}\right)\right. \\
-2 \cot 2 x_{1}+4 \tan x_{1} \\
+2 \tan \left(x_{1}-x_{2}\right)+\tan \left(x_{1}+x_{2}\right), \\
4 \cot \left(x_{1}-x_{2}\right)-4 \cot x_{2}-4 \cot \left(x_{1}+x_{2}\right) \\
-2 \cot 2 x_{2}-2 \tan \left(x_{1}-x_{2}\right)+5 \tan x_{2} \\
\left.\quad+\tan \left(x_{1}+x_{2}\right)\right) \\
\left(\widetilde{C}: x_{1}>0, x_{2}>x_{1}, x_{1}+x_{2}<\frac{\pi}{2}\right)\end{array}$ \\
\hline
\end{tabular}

TAble 3 (Continued $\left.{ }^{3}\right)$. 


\begin{tabular}{|c|c|}
\hline$H \curvearrowright G / K$ & $(\widetilde{C})$ \\
\hline $\begin{array}{c}\rho_{11}(\operatorname{Spin}(10) \cdot U(1)) \curvearrowright \\
E_{6} / \operatorname{Spin}(10) \cdot U(1)\end{array}$ & $\begin{array}{l}X_{\left(x_{1}, x_{2}\right)}=\left(-8 \cot x_{1}-2 \cot 2 x_{1}\right. \\
+6 \tan \left(x_{1}-x_{2}\right)+5 \tan \left(x_{1}+x_{2}\right) \\
\quad-2 \cot 2 x_{2}-6 \tan \left(x_{1}-x_{2}\right) \\
\left.\quad+9 \tan x_{2}+5 \tan \left(x_{1}+x_{2}\right)\right) \\
\left(\widetilde{C}: x_{1}>0, x_{2}>0, x_{1}+x_{2}<\frac{\pi}{2}\right)\end{array}$ \\
\hline $\begin{array}{c}\rho_{12}(\operatorname{Spin}(10) \cdot U(1)) \curvearrowright \\
E_{6} / \operatorname{Spin}(10) \cdot U(1)\end{array}$ & $\begin{array}{c}X_{\left(x_{1}, x_{2}\right)}=\left(-6 \cot x_{1}-\cot \left(x_{1}-x_{2}\right)-\cot \left(x_{1}+x_{2}\right)\right. \\
2 \tan x_{1}+5 \tan \left(x_{1}-x_{2}\right) \\
+4 \tan \left(x_{1}+x_{2}\right)+2 \tan 2 x_{1} \\
\cot \left(x_{1}-x_{2}\right)-6 \cot x_{2}-\cot \left(x_{1}+x_{2}\right) \\
-5 \tan \left(x_{1}-x_{2}\right)+3 \tan x_{2} \\
\left.+4 \tan \left(x_{1}+x_{2}\right)+2 \tan 2 x_{2}\right) \\
\left(\widetilde{C}: x_{1}>0, x_{2}<\frac{\pi}{4}, x_{2}>x_{1}\right) \\
\end{array}$ \\
\hline$S p(4) \curvearrowright E_{6} / F_{4}$ & $\begin{array}{c}X_{\left(x_{1}, x_{2}\right)}=\left(-8 \cot 2 x_{1}-4 \cot \left(x_{1}-\sqrt{3} x_{2}\right)-4 \cot \left(x_{1}+\sqrt{3} x_{2}\right)\right. \\
+8 \tan 2 x_{1}+4 \tan \left(x_{1}-\sqrt{3} x_{2}\right)+4 \tan \left(x_{1}+\sqrt{3} x_{2}\right) \\
4 \sqrt{3} \cot \left(x_{1}-\sqrt{3} x_{2}\right)-4 \sqrt{3} \cot \left(x_{1}+\sqrt{3} x_{2}\right) \\
\left.-4 \sqrt{3} \tan \left(x_{1}-\sqrt{3} x_{2}\right)+4 \sqrt{3} \tan \left(x_{1}+\sqrt{3} x_{2}\right)\right) \\
\left(\widetilde{C}: 0<x_{1}<\frac{\pi}{4}, \frac{x_{1}}{\sqrt{3}}<x_{2}<\frac{x_{1}}{\sqrt{3}}+\frac{\pi}{2 \sqrt{3}},-\frac{x_{1}}{\sqrt{3}}<x_{2}<-\frac{x_{1}}{\sqrt{3}}+\frac{\pi}{2 \sqrt{3}}\right)\end{array}$ \\
\hline$\rho_{13}\left(F_{4}\right) \curvearrowright E_{6} / F_{4}$ & $\begin{aligned} X_{\left(x_{1}, x_{2}\right)} & =\left(-16 \cot 2 x_{1}+8 \tan \left(x_{1}-\sqrt{3} x_{2}\right)+8 \tan \left(x_{1}+\sqrt{3} x_{2}\right)\right. \\
& \left.-8 \sqrt{3} \tan \left(x_{1}-\sqrt{3} x_{2}\right)+8 \sqrt{3} \tan \left(x_{1}+\sqrt{3} x_{2}\right)\right) \\
& \left(\widetilde{C}: x_{1}>0, x_{1}-\sqrt{3} x_{2}<\frac{\pi}{2}, x_{1}+\sqrt{3} x_{2}<\frac{\pi}{2}\right)\end{aligned}$ \\
\hline$\rho_{14}(S O(4)) \curvearrowright G_{2} / S O(4)$ & $\begin{array}{rl}X_{\left(x_{1}, x_{2}\right)}= & \left(-2 \cot 2 x_{1}+3 \tan \left(3 x_{1}-\sqrt{3} x_{2}\right)+\tan \left(x_{1}-\sqrt{3} x_{2}\right)\right. \\
& +\tan \left(x_{1}+\sqrt{3} x_{2}\right)+3 \tan \left(3 x_{1}+\sqrt{3} x_{2}\right) \\
-2 \sqrt{3} \cot 2 & 2 \sqrt{3} x_{2}-\sqrt{3} \tan \left(3 x_{1}-\sqrt{3} x_{2}\right)-\sqrt{3} \tan \left(x_{1}-\sqrt{3} x_{2}\right) \\
+ & \left.\sqrt{3} \tan \left(x_{1}+\sqrt{3} x_{2}\right)+\sqrt{3} \tan \left(3 x_{1}+\sqrt{3} x_{2}\right)\right) \\
& \left(\widetilde{C}: x_{1}>0, x_{2}>0, \sqrt{3} x_{1}+x_{2}<\frac{\pi}{2 \sqrt{3}}\right)\end{array}$ \\
\hline$\rho_{15}(S O(4)) \curvearrowright G_{2} / S O(4)$ & $\begin{aligned} X_{\left(x_{1}, x_{2}\right)}= & \left(-2 \cot 2 x_{1}+3 \tan \left(3 x_{1}-\sqrt{3} x_{2}\right)+\tan \left(x_{1}-\sqrt{3} x_{2}\right)\right. \\
& +\tan \left(x_{1}+\sqrt{3} x_{2}\right)+3 \tan \left(3 x_{1}+\sqrt{3} x_{2}\right) \\
-2 \sqrt{3} \cot 2 & \sqrt{3} x_{2}-\sqrt{3} \tan \left(3 x_{1}-\sqrt{3} x_{2}\right)-\sqrt{3} \tan \left(x_{1}-\sqrt{3} x_{2}\right) \\
+ & \left.\sqrt{3} \tan \left(x_{1}+\sqrt{3} x_{2}\right)+\sqrt{3} \tan \left(3 x_{1}+\sqrt{3} x_{2}\right)\right) \\
& \left(\widetilde{C}: x_{1}>0, x_{2}>0, \sqrt{3} x_{1}+x_{2}<\frac{\pi}{2 \sqrt{3}}\right)\end{aligned}$ \\
\hline
\end{tabular}

Table 3 (COntinued ${ }^{4}$ ). 


\begin{tabular}{|c|c|}
\hline$H \curvearrowright G / K$ & $X \quad(\widetilde{C})$ \\
\hline$\rho_{16}\left(G_{2}\right) \curvearrowright\left(G_{2} \times G_{2}\right) / G_{2}$ & $X_{\left(x_{1}, x_{2}\right)}=2\left(-2 \cot 2 x_{1}+3 \tan \left(3 x_{1}-\sqrt{3} x_{2}\right)+\tan \left(x_{1}-\sqrt{3} x_{2}\right)\right.$ \\
& $+\tan \left(x_{1}+\sqrt{3} x_{2}\right)+3 \tan \left(3 x_{1}+\sqrt{3} x_{2}\right)$, \\
& $-2 \sqrt{3} \cot 2 \sqrt{3} x_{2}-\sqrt{3} \tan \left(3 x_{1}-\sqrt{3} x_{2}\right)-\sqrt{3} \tan \left(x_{1}-\sqrt{3} x_{2}\right)$ \\
& $\left.+\sqrt{3} \tan \left(x_{1}+\sqrt{3} x_{2}\right)+\sqrt{3} \tan \left(3 x_{1}+\sqrt{3} x_{2}\right)\right)$ \\
& $\left(\widetilde{C}: x_{1}>0, x_{2}>0, \sqrt{3} x_{1}+x_{2}<\frac{\pi}{2 \sqrt{3}}\right)$ \\
& \\
\hline$S U(2)^{4} \curvearrowright\left(G_{2} \times G_{2}\right) / G_{2}$ & $X_{\left(x_{1}, x_{2}\right)}=\left(-2 \cot 2 x_{1}-3 \cot \left(3 x_{1}-\sqrt{3} x_{2}\right)-\cot \left(x_{1}-\sqrt{3} x_{2}\right)\right.$ \\
& $-\cot \left(x_{1}+\sqrt{3} x_{2}\right)-3 \cot \left(3 x_{1}+\sqrt{3} x_{2}\right)+2 \tan 2 x_{1}$ \\
& $+3 \tan \left(3 x_{1}-\sqrt{3} x_{2}\right)+\tan \left(x_{1}-\sqrt{3} x_{2}\right)$ \\
& $+\tan \left(x_{1}+\sqrt{3} x_{2}\right)+3 \tan \left(3 x_{1}+\sqrt{3} x_{2}\right)$, \\
& $\left.\sqrt{3} x_{2}\right)+\sqrt{3} \cot \left(x_{1}-\sqrt{3} x_{2}\right)-\sqrt{3} \cot \left(x_{1}+\sqrt{3} x_{2}\right)$ \\
& $-\sqrt{3} \cot \left(3 x_{1}-\sqrt{3} x_{2}\right)-2 \sqrt{3} \cot 2 \sqrt{3} x_{2}-\sqrt{3} \tan \left(3 x_{1}-\sqrt{3} x_{2}\right)$ \\
& $-\sqrt{3} \cot \left(3 x_{1}+\sqrt{3} \tan \left(x_{1}-\sqrt{3} x_{2}\right)+\sqrt{3} \tan \left(x_{1}+\sqrt{3} x_{2}\right)\right.$ \\
& $\left.+\sqrt{3} \tan \left(3 x_{1}+\sqrt{3} x_{2}\right)+2 \sqrt{3} \tan 2 \sqrt{3} x_{2}\right)$ \\
& $\left(\widetilde{C}: x_{1}>0, x_{2}<\frac{\pi}{4 \sqrt{3}}, x_{2}>\sqrt{3} x_{1}\right)$ \\
\hline
\end{tabular}

TABle $3\left(\right.$ CONTINUED $\left.^{5}\right)$.

\section{REFERENCES}

[BV] J. Berndt And L. VAnhecke, Curvature-adapted submanifolds, Nihonkai Math. J., 3 (1992), pp. $177-185$.

[BCO] J. Berndt, S. Console, and C. Olmos, Submanifolds and holonomy, Research Notes in Mathematics 434, CHAPMAN \& HALL/CRC Press, Boca Raton, London, New York, Washington, 2003.

[B] N. Bourbaki, Groupes et algébres de Lie, Chapitre 4,5 et 6, Hermann, 1968.

[Ch] U. Christ, Homogeneity of equifocal submanifolds, J. Differential Geometry, 62 (2002), pp. 1-15.

[Co] L. Conlon, Remarks on commuting involutions, Proc. Amer. Math. Soc., 22 (1969), pp. 255-257.

[GT] O. Goertsches and G. Thorbergsson, On the Geometry of the orbits of Hermann actions, Geom. Dedicata, 129 (2007), pp. 101-118.

[He] S. Helgason, Differential geometry, Lie groups and symmetric spaces, Academic Press, New York, 1978.

[Ha] R. S. Hamilton, Three-manifolds with positive Ricci curvature, J. Differential Geom., 17 (1982), pp. 255-306.

[Hu1] G. Huisken, Flow by mean curvature of convex surfaces into spheres, J. Differential Geom., 20 (1984), pp. 237-266.

[Hu2] G. Huisken, Contracting convex hypersurfaces in Riemannian manifolds by their mean curvature, Invent. math., 84 (1986), pp. 463-480.

[HLO] E. Heintze, X. Liu, AND C. Olmos, Isoparametric submanifolds and a Chevalley-type restriction theorem, Integrable systems, geometry, and topology, pp. 151-190, AMS/IP Stud. Adv. Math. 36, Amer. Math. Soc., Providence, RI, 2006.

[HOT] E. Heintze, C. Olmos, And G. Thorbergsson, Submanifolds with constant principal curvatures and normal holonomy groups, Intern. J. Math., 2 (1991), pp. 167-175.

[HPTT] E. Heintze, R. S. Palais, C. L. Terng, and G. Thorbergsson, Hyperpolar actions on symmetric spaces, Geometry, topology and physics for Raoul Bott (ed. S. T. Yau), Conf. Proc. Lecture Notes Geom. Topology 4, Internat. Press, Cambridge, MA, 1995, pp. $214-245$.

[HTST] D. Hirohashi, H. Tasaki, H. Song, and R. Takagi, Minimal orbits of the isotropy groups of symmetric spaces of compact type, Differential Goem. and its Appl., 13 (2000), pp. 167-177. 
[Koi1] N. KOIKE, On proper Fredholm submanifolds in a Hilbert space arising from subma-nifolds in a symmetric space, Japan. J. Math., 28 (2002), pp. 61-80.

[Koi2] N. KoIKE, Actions of Hermann type and proper complex equifocal submanifolds, Osaka J. Math., 42 (2005), pp. 599-611.

[Kol] A. Kollross, A Classification of hyperpolar and cohomogeneity one actions, Trans. Amer. Math. Soc., 354 (2001), pp. 571-612.

[LT] X. LIU AND C. L. TERng, The mean curvature flow for isoparametric submanifolds, arXiv:math.DG/0706.3550v1.

[P] R. S. PAlais, Morse theory on Hilbert manifolds, Topology, 2 (1963), pp. 299-340.

[PT] R. S. PAlais And C. L. TERng, Critical point theory and submanifold geometry, Lecture Notes in Math., 1353, Springer, Berlin, 1988.

[S] G. Schwarz, Smooth functions invariant under the action of a compact Lie group, Topology, 14 (1975), pp. 63-68.

[T1] C. L. Terng, Isoparametric submanifolds and their Coxeter groups, J. Differential Geometry , 21 (1985), pp. 79-107.

[T2] C. L. Terng, Proper Fredholm submanifolds of Hilbert space, J. Differential Geometry, 29 (1989), pp. 9-47.

[TT] C. L. Terng AND G. Thorbergsson, Submanifold geometry in symmetric spaces, J. Differential Geometry, 42 (1995), pp. 665-718.

[Z] X. P. ZHU, Lectures on mean curvature flows, Studies in Advanced Math., AMS/IP, 2002. 
